\title{
TOWERS OF GRADED SUPERALGEBRAS CATEGORIFY THE TWISTED HEISENBERG DOUBLE
}

\author{
DANIELE ROSSO AND ALISTAIR SAVAGE
}

\begin{abstract}
АвsтRAct. We show that the Grothendieck groups of the categories of finitely-generated graded supermodules and finitely-generated projective graded supermodules over a tower of graded superalgebras satisfying certain natural conditions give rise to twisted Hopf algebras that are twisted dual. Then, using induction and restriction functors coming from such towers, we obtain a categorification of the twisted Heisenberg double, introduced in [RS], and its Fock space representation. We show that towers of wreath product algebras (in particular, the tower of Sergeev superalgebras) and the tower of nilCoxeter graded superalgebras satisfy our axioms. In the latter case, one obtains a categorification of the quantum Weyl algebra.
\end{abstract}

\section{Contents}

1. Introduction 1

2. The twisted Heisenberg double 3

3. Supermodules over graded superalgebras 5

4. Towers of graded superalgebras 10

5. Categorification of the twisted Heisenberg double and its Fock space 13

6. Frobenius graded superalgebras 16

7. Towers of wreath product algebras 20

8. The tower of nilCoxeter graded superalgebras 24

References $\quad 28$

\section{INTRODUCTION}

The Heisenberg double is an associative algebra constructed in a natural way from a Hopf algebra. As a $\mathbb{k}$-module, the Heisenberg double $\mathfrak{h}\left(\mathrm{H}^{+}, \mathrm{H}^{-}\right)$of a Hopf algebra $\mathrm{H}^{+}$ over a ring $\mathbb{k}$, with dual $H^{-}$, is isomorphic to $H^{+} \otimes_{\mathbb{k}} H^{-}$. The factors $H^{ \pm}$are subalgebras, and there is an explicit commutation relation between the two factors. The Heisenberg double can be viewed naturally as the subalgebra of $\mathrm{End}_{\mathrm{k}} \mathrm{H}^{+}$generated by left multiplication by $\mathrm{H}^{+}$and the left regular action of $\mathrm{H}^{-}$on $\mathrm{H}^{+}$. From this point of view, one sees that the Heisenberg double has a natural representation on $\mathrm{H}^{+}$, called the Fock space

2010 Mathematics Subject Classification. 16D90, 17A70, 16 T05.

Key words and phrases. Twisted Heisenberg double, tower of algebras, graded superalgebra, Frobenius graded superalgebra, categorification, twisted Hopf algebra, Fock space, nilCoxeter algebra.

The second author was supported by a Discovery Grant from the Natural Sciences and Engineering Research Council of Canada. The first author was supported by the Centre de Recherches Mathématiques and the Discovery Grant of the second author. 
representation. The most well-known example is when $H^{+}$and $H^{-}$are both the self-dual Hopf algebra of symmetric functions, in which case the Heisenberg double is the classical infinite-dimensional Heisenberg algebra.

In [RS], the authors introduced a twisted version of the Heisenberg double. This involved replacing Hopf algebras by twisted Hopf algebras (see Section 2) and the Hopf pairing between $\mathrm{H}^{+}$and $\mathrm{H}^{-}$by a twisted Hopf pairing (see Definition 2.1). If these data satisfy a certain natural compatibility condition (see Definition 2.4), one can construct the twisted Heisenberg double, which also has a natural Fock space representation. In the case that the twistings are trivial, one recovers the usual notion of the Heisenberg double.

One of the main motivations for the introduction of the twisted Heisenberg double in [RS] was applications to categorification. A conjectural categorification of the infinitedimensional Heisenberg algebra itself was first described by Khovanov in [Kho] (see also [LS13]). Since then, the Heisenberg algebra has been categorified in several ways. We refer the reader to the expository paper [LS12] for an overview of these approaches. Motivated by these constructions and results on dual Hopf algebras obtained from towers of algebras (see [BL09]), a weak categorification of the more general Heisenberg double was developed in [SY15]. The dual Hopf algebras $H^{ \pm}$correspond to the Grothendieck groups of the categories of finitely-generated modules and finitely-generated projective modules of a tower of algebras satisfying some natural conditions. Then, using certain induction and restriction functors, one obtains a categorification of the associated Heisenberg double and its Fock space representation.

The goal of the current paper is to extend the results of [SY15] to the setting of towers of graded superalgebras. Since graded algebras and superalgebras are both ubiquitous in the categorification literature, the extension to this setting is a natural one. Typically, passing to graded algebras results, on the level of Grothendieck groups, in a quantum deformation. In the current paper, it results in the move from Hopf algebras to twisted Hopf algebras, and from the Heisenberg double to the twisted Heisenberg double. On the other hand, generalizing from algebras to superalgebras introduces extra structure on the Grothendieck groups coming from the parity shift functor. After background on the twisted Heisenberg double in Section 2 and on categories of supermodules over graded superalgebras in Section 3, we introduce our main objects of study, towers of graded superalgebras, in Section 4. In Section 5, we present our main results, Theorem 5.7 and Corollary 5.8, that so-called strong compatible towers of graded superalgebras give rise to a (weak) categorification of the twisted Heisenberg double and its Fock space representation.

We also include in this paper several applications of our main result. First, in Section 6, we recall some facts about Frobenius graded superalgebras, using a slightly more general definition of these objects than is often considered in the literature. We show that towers of Frobenius graded superalgebras automatically satisfy one of the important conditions of a strong tower. Namely, induction is conjugate shifted right adjoint to restriction (see Proposition 6.7). In Section 7, we discuss a large class of examples of strong compatible towers, namely, towers of wreath product algebras (see Corollary 7.6). This class includes the tower of Sergeev superalgebras and the algebras considered in the Heisenberg categorification of [CL12]. We conclude, in Section 8, with a discussion of the tower 
of nilCoxeter algebras, viewed as graded superalgebras. This tower is also strong and compatible, and yields a categorification of the quantum Weyl algebra.

Note on the arXiv version. For the interested reader, the tex file of the arXiv version of this paper includes hidden details of some straightforward computations and arguments that are omitted in the pdf file. These details can be displayed by switching the details toggle to true in the tex file and recompiling.

Notation. We let $\mathbb{N}$ and $\mathbb{N}_{+}$denote the set of nonnegative and positive integers respectively. We let $\mathbb{Z}_{2}=\mathbb{Z} / 2 \mathbb{Z}$ be the ring of integers mod 2 . We let $\mathbb{F}$ be a field of characteristic not equal to two. By a slight abuse of terminology, we will use the terms module and representation interchangeably.

Acknowledgements. The authors would like to thank A. Licata, J. Sussan, and O. Yacobi for useful conversations.

\section{The twisted Heisenberg double}

In this section, we review the concept, introduced in [RS], of the twisted Heisenberg double and its Fock space representation. We fix a commutative ring $\mathbb{k}$ and all algebras, coalgebras, bialgebras and Hopf algebras will be over $\mathbb{k}$. We will denote the multiplication, comultiplication, unit, counit and antipode of a Hopf algebra by $\nabla, \Delta, \eta, \varepsilon$ and $S$ respectively. We write the product $\nabla$ as juxtaposition when this will not cause confusion, and use Sweedler notation

$$
\Delta(a)=\sum_{(a)} a_{1} \otimes a_{2}
$$

for coproducts. All tensor products are over $\mathbb{k}$ unless otherwise indicated.

Let $(\Lambda,+)$ be a commutative monoid isomorphic (as a monoid) to $\mathbb{N}^{r}$. Consider $H=$ $\bigoplus_{\lambda \in \Lambda} H_{\lambda}$, where each $H_{\lambda}, \lambda \in \Lambda$, is finitely generated and free as a $\mathbb{k}$-module, such that $(H, \nabla, \varepsilon)$ is a $\Lambda$-graded algebra and $(H, \Delta, \eta)$ is a $\Lambda$-graded coalgebra. This means, in particular, that the following conditions are satisfied:

$$
\begin{gathered}
\nabla\left(H_{\lambda} \otimes H_{\mu}\right) \subseteq H_{\lambda+\mu}, \quad \Delta\left(H_{\lambda}\right) \subseteq \bigoplus_{\mu+v=\lambda} H_{\mu} \otimes H_{v}, \quad \lambda, \mu, v \in \Lambda, \\
\eta(\mathbb{k}) \subseteq H_{0}, \quad \varepsilon\left(H_{\lambda}\right)=0 \text { for } \lambda \in \Lambda \backslash\{0\} .
\end{gathered}
$$

Fix $c \in \mathbb{k}^{\times}$, and let $\chi=\left(\chi^{\prime}, \chi^{\prime \prime}\right)$ be a pair of biadditive maps $\chi^{\prime}, \chi^{\prime \prime}: \Lambda \times \Lambda \rightarrow \mathbb{Z}$. Define a new multiplication $*_{\chi}$ on $H \otimes H$ by the condition that, for homogeneous elements $a_{i}, b_{i} \in H$, $i=1,2$, we have

$$
\left(a_{1} \otimes a_{2}\right) *_{\chi}\left(b_{1} \otimes b_{2}\right)=c^{\chi^{\prime}\left(\left|a_{2}\right|,\left|b_{1}\right|\right)+\chi^{\prime \prime}\left(\left|a_{1}\right|,\left|b_{2}\right|\right)} a_{1} b_{1} \otimes a_{2} b_{2},
$$

where $|a|$ denotes the degree of a homogeneous element $a \in H$. (Whenever we write an expression involving $|a|$ for some $a \in H$, we implicitly assume that $a$ is homogeneous.) Since $\chi^{\prime}, \chi^{\prime \prime}$ are biadditive, $*_{\chi}$ is associative, and we denote by $(H \otimes H)_{\chi}$ this twisted associative algebra structure.

We say that $H$ is a $\Lambda$-graded connected twisted bialgebra, or, more precisely, a $(c, \chi)$-bialgebra if $H_{0}=\mathbb{k} 1_{H}$ and

$$
\Delta: H \rightarrow(H \otimes H)_{\chi}
$$


is an algebra homomorphism. A $\Lambda$-graded connected twisted bialgebra is always a twisted Hopf algebra, that is, there exists a $\mathbb{k}$-linear map $S: H \rightarrow H$ called the antipode that satisfies the condition

$$
\nabla(\mathrm{Id} \otimes S) \Delta=\eta \varepsilon=\nabla(S \otimes \mathrm{Id}) \Delta .
$$

Thus we will also call such an object a $\Lambda$-graded connected $(c, \chi)$-Hopf algebra, or simply a $(c, \chi)$-Hopf alegbra. When we wish to leave the data $(c, \chi)$ implied, we refer to it simply as a twisted Hopf algebra. We will write $\left(q, \chi^{\prime}, \chi^{\prime \prime}\right)$ instead of $\left(q,\left(\chi^{\prime}, \chi^{\prime \prime}\right)\right)$ when we wish to make the components of $\chi$ explicit.

Definition 2.1 (Twisted Hopf pairing). Suppose $H$ and $H^{\prime}$ are twisted Hopf algebras and $\gamma=\left(\gamma^{\prime}, \gamma^{\prime \prime}\right)$ is a pair of biadditive maps $\gamma^{\prime}, \gamma^{\prime \prime}: \Lambda \times \Lambda \rightarrow \mathbb{Z}$. Then a $(c, \gamma)$-twisted Hopf pairing of $H$ and $H^{\prime}$ is a bilinear map $\langle-,-\rangle: H \times H^{\prime} \rightarrow \mathbb{k}$ such that $\left.\langle-,-\rangle\right|_{H_{\lambda} \times H_{\mu}^{\prime}} \equiv 0$ when $\lambda, \mu \in \Lambda, \lambda \neq \mu$, and

$$
\begin{gathered}
\langle x y, a\rangle=c^{\gamma^{\prime}(|x|,|y|)}\langle x \otimes y, \Delta(a)\rangle, \\
\langle x, a b\rangle=c^{\gamma^{\prime \prime}(|a|,|b|)}\langle\Delta(x), a \otimes b\rangle, \\
\left\langle 1_{H}, a\right\rangle=\varepsilon(a), \quad\left\langle x, 1_{H^{\prime}}\right\rangle=\varepsilon(x),
\end{gathered}
$$

for all homogeneous $x, y \in H, a, b \in H^{\prime}$, where we define

$$
\langle-,-\rangle:(H \otimes H) \otimes\left(H^{\prime} \otimes H^{\prime}\right) \rightarrow \mathbb{k}, \quad\langle x \otimes y, a \otimes b\rangle=\langle x, a\rangle\langle y, b\rangle, \quad x, y \in H, a, b \in H^{\prime} .
$$

We will write $\left(c, \gamma^{\prime}, \gamma^{\prime \prime}\right)$ instead of $\left(c,\left(\gamma^{\prime}, \gamma^{\prime \prime}\right)\right)$ when we wish to make the components of $\gamma$ explicit. A $(1, \gamma)$-twisted Hopf pairing (equivalently, a $(c, 0,0)$-twisted Hopf pairing) is simply called a Hopf pairing.

Definition 2.2 (Dual pair). We say that $\left(H^{+}, H^{-}\right)$is a $(c, \gamma)$-dual pair of twisted Hopf algebras if $\mathrm{H}^{+}$and $\mathrm{H}^{-}$are both twisted Hopf algebras, and there exists a $(c, \gamma)$-twisted Hopf pairing $\langle-,-\rangle: H^{-} \times H^{+} \rightarrow \mathbb{k}$ such that $\left.\langle-,-\rangle\right|_{H_{\lambda}^{-} \times H_{\lambda}^{+}}, \lambda \in \Lambda$, is a perfect pairing. When we wish to leave the data $(c, \gamma)$ implicit, we say that the pair $\left(H^{+}, H^{-}\right)$is twisted dual.

For a biadditive map $\zeta: \Lambda \times \Lambda \rightarrow \mathbb{Z}$, we define

$$
\zeta^{T}: \Lambda \times \Lambda \rightarrow \mathbb{Z}, \quad \zeta^{T}(\lambda, \mu)=\zeta(\mu, \lambda), \quad \lambda, \mu \in \Lambda .
$$

Lemma 2.3 ([RS, Lem. 2.8]). Suppose $\left(H^{+}, H^{-}\right)$is a $(c, \gamma)$-dual pair of twisted Hopf algebras and $\mathrm{H}^{+}$is a $(c, \chi)$-Hopf algebra. Then $\mathrm{H}^{-}$is a $(c, \xi)$-Hopf algebra, where

$$
\xi=\left(\xi^{\prime}, \xi^{\prime \prime}\right), \quad \xi^{\prime}=\left(\chi^{\prime}\right)^{T}+\gamma^{\prime}-\left(\gamma^{\prime \prime}\right)^{T}, \quad \xi^{\prime \prime}=\chi^{\prime \prime}+\gamma^{\prime}-\gamma^{\prime \prime} .
$$

For the remainder of this section, we fix a $(c, \gamma)$-dual pair $\left(H^{+}, H^{-}\right)$of twisted Hopf algebras, where $\mathrm{H}^{+}$is a $(c, \chi)$-Hopf algebra and $H^{-}$is a $(c, \xi)$-Hopf algebra, with $\xi$ given by (2.2).

Any $a \in H^{+}$defines an element ${ }^{L} a \in$ End $_{\mathbb{k}} H^{+}$by left multiplication. Similarly, any $x \in H^{-}$defines an element ${ }^{R} x \in$ End $_{\mathbb{k}} H^{-}$by right multiplication, whose adjoint ${ }^{R} x^{*}$ is an element of $\operatorname{End}_{\mathbb{k}} H^{+}$. (In the case that $H^{+}$or $H^{-}$is commutative, we often omit the superscript $L$ or $R$.) In this way we have $\mathbb{k}$-algebra homomorphisms

$$
\begin{aligned}
& H^{+} \hookrightarrow \operatorname{End}_{\mathbb{k}} H^{+}, \quad a \mapsto{ }^{L} a, \\
& H^{-} \hookrightarrow \operatorname{End}_{\mathbb{k}} H^{+}, \quad x \mapsto{ }^{R} x^{*} .
\end{aligned}
$$


The action of $\mathrm{H}^{-}$on $\mathrm{H}^{+}$given by (2.4) is called the left regular action. The maps (2.3) and (2.4) are both injective. For $x \in H^{-}$and $a, b \in H^{+}$, we have, by [RS, Lem. 3.2] and (2.2),

$$
R x^{*}(a b)=\sum_{(x)} c^{\gamma^{\prime}\left(|a|-\left|x_{1}\right|,\left|x_{2}\right|\right)+\gamma^{\prime}\left(|b|-\left|x_{2}\right|,\left|x_{1}\right|\right)+\gamma^{\prime \prime}\left(\left|x_{1}\right|,\left|x_{2}\right|\right)+\chi^{\prime}\left(\left|x_{1}\right|,|b|-\left|x_{2}\right|\right)+\chi^{\prime \prime}\left(|a|-\left|x_{1}\right|,\left|x_{2}\right|\right) R} x_{1}^{*}(a)^{R} x_{2}^{*}(b) .
$$

Definition 2.4 (Compatible dual pair). We say that the $(c, \gamma)$-dual pair $\left(H^{+}, H^{-}\right)$is compatible if there is a choice of $\chi$ such that $H^{+}$is a $(c, \chi)$-Hopf algebra and

$$
\chi^{\prime}=-\left(\gamma^{\prime}\right)^{T} \text {. }
$$

If the pair $\left(H^{+}, H^{-}\right)$is compatible and we choose $\chi$ to satisfy (2.6), then (2.5) implies that, for all $x \in H^{-}$and $a \in H^{+}$, we have,

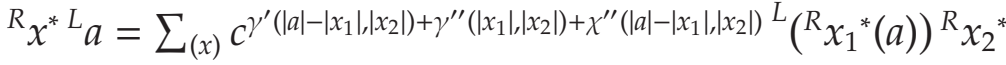

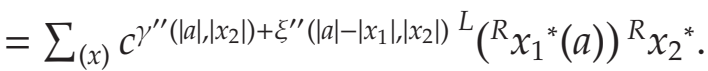

Definition 2.5 (The twisted Heisenberg double). Suppose the pair $\left(H^{+}, H^{-}\right)$is compatible and we choose $\chi$ to satisfy (2.6). We define the twisted Heisenberg double $\mathfrak{h}\left(\mathrm{H}^{+}, \mathrm{H}^{-}\right)$of $\mathrm{H}^{+}$ as follows. We set $\mathfrak{h}\left(H^{+}, H^{-}\right)=H^{+} \otimes H^{-}$as $\mathbb{k}$-modules, and we write $a \# x$ for $a \otimes x, a \in H^{+}$, $x \in H^{-}$, viewed as an element of $\mathfrak{h}\left(H^{+}, H^{-}\right)$. Multiplication is given by

$$
\begin{aligned}
(a \# x)(b \# y): & :=\sum_{(x)} c^{\gamma^{\prime \prime}\left(|b|,\left|x_{2}\right|\right)+\xi^{\prime \prime}\left(|b|-\left|x_{1}\right|,\left|x_{2}\right|\right)} a^{R} x_{1}^{*}(b) \# x_{2} y \\
& =\sum_{(x),(b)} \gamma^{\gamma^{\prime \prime}\left(|b|,\left|x_{2}\right|\right)+\xi^{\prime \prime}\left(|b|-\left|x_{1}\right|,\left|x_{2}\right|\right)+\gamma^{\prime}\left(\left|b_{1}\right|,\left|b_{2}\right|\right)}\left\langle x_{1}, b_{2}\right\rangle a b_{1} \# x_{2} y,
\end{aligned}
$$

where the second equality follows from [RS, Lem. 3.1]. We will often view $\mathrm{H}^{+}$and $\mathrm{H}^{-}$as subalgebras of $\mathfrak{h}\left(H^{+}, H^{-}\right)$via the maps $a \mapsto a \# 1$ and $x \mapsto 1 \# x$ for $a \in H^{+}$and $x \in H^{-}$. Then we have $a x=a \# x$. When the context is clear, we will simply write $\mathfrak{h}$ for $\mathfrak{h}\left(H^{+}, H^{-}\right)$.

An element $v$ of an $\mathfrak{h}$-module $V$ is called a lowest weight (resp. highest weight) vacuum vector if $\mathbb{k} v \cong \mathbb{k}$, as $\mathbb{k}$-modules, and $H_{\lambda}^{-} v=0$ (resp. $H_{\lambda}^{+} v=0$ ) for all $\lambda \neq 0$. The algebra $\mathfrak{h}$ has a natural (left) representation on $H^{+}$given by

$$
(a \# x)(b)=a^{R} x^{*}(b), \quad a, b \in H^{+}, x \in H^{-} .
$$

We call this the lowest weight Fock space representation of $\mathfrak{h}$ and denote it by $\mathcal{F}=\mathcal{F}\left(H^{+}, H^{-}\right)$. Note that this representation is generated by the lowest weight vacuum vector $1 \in H^{+}$. By [RS, Th. 4.3(d)], the action of $\mathfrak{h}$ on $\mathcal{F}$ is faithful. Thus, we may view $\mathfrak{h}$ as the subalgebra of End $\mathbb{k}_{\mathrm{k}} H^{+}$generated by ${ }^{L} a, a \in H^{+}$, and ${ }^{R} x^{*}, x \in H^{-}$. If $X^{+}$is a $\Lambda$-graded subalgebra of $H^{+}$ that is invariant under the left regular action of $\mathrm{H}^{-}$on $\mathrm{H}^{+}$, then $\mathrm{X}^{+} \# \mathrm{H}^{-}$is a subalgebra of h acting naturally on $X^{+}$.

\section{Supermodules over graded SUPERALGEBras}

In this section, we collect some facts about categories of supermodules over graded superalgebras and the Grothendieck groups of these categories. Recall that $\mathbb{F}$ is an arbitrary field of characteristic not equal to two. 
3.1. Supermodule categories and homomorphism spaces. Let $B$ be a graded (more precisely, $\mathbb{N}$-graded) superalgebra over $\mathbb{F}$. By this we mean that

$$
B \cong \bigoplus_{i \in \mathbb{N}, \epsilon \in \mathbb{Z}_{2}} B_{i, \epsilon} \quad \text { with } \quad B_{i, \epsilon} B_{j, \tau} \subseteq B_{i+j, \epsilon+\tau}, \quad i, j \in \mathbb{N}, \epsilon, \tau \in \mathbb{Z}_{2} .
$$

If $B$ is a graded superalgebra, a graded $B$-supermodule $M$ is analogously a $\left(\mathbb{Z} \times \mathbb{Z}_{2}\right)$-graded vector space over $\mathbb{F}$ such that

$$
B_{i, \epsilon} M_{j, \tau} \subseteq M_{i+j, \epsilon+\tau} \quad \text { for all } i \in \mathbb{N}, j \in \mathbb{Z}, \epsilon, \tau \in \mathbb{Z}_{2} \text {. }
$$

(Throughout the paper, all (super)modules are left (super)modules unless otherwise specified.) If $v$ is a homogeneous element in a $\mathbb{Z}$-graded (resp. $\mathbb{Z}_{2}$-graded) vector space, we will denote by $|v|$ (resp. $\bar{v}$ ) its degree. Whenever we write an expression involving degrees of elements, we will implicitly assume that such elements are homogeneous.

For $M, N$ two $\left(\mathbb{Z} \times \mathbb{Z}_{2}\right)$-graded vector spaces over $\mathbb{F}$, we define a $\left(\mathbb{Z} \times \mathbb{Z}_{2}\right)$-grading on the space $\operatorname{HOM}_{\mathbb{F}}(M, N)$ of all $\mathbb{F}$-linear maps by setting $\operatorname{HOM}_{\mathbb{F}}(M, N)_{i, \epsilon}$ to be the subspace of all homogeneous maps of degree $(i, \epsilon)$. That is,

$$
\operatorname{HOM}_{\mathbb{F}}(M, N)_{i, \epsilon}:=\left\{\alpha \in \operatorname{HOM}_{\mathbb{F}}(M, N) \mid \alpha\left(M_{j, \tau}\right) \subseteq N_{i+j, \epsilon+\tau} \forall j \in \mathbb{Z}, \tau \in \mathbb{Z}_{2}\right\} .
$$

If $M, N$ are two graded $B$-supermodules, then we define the $\mathbb{F}$-vector space

$$
\operatorname{Hom}_{B}(M, N):=\left\{f \in \operatorname{HOM}_{\mathbb{F}}(M, N)_{0,0} \mid f(b m)=b f(m) \forall b \in B, m \in M\right\} .
$$

For $B$ a finite-dimensional graded superalgebra, let $B$-grmod denote the category of finitely-generated graded left $B$-supermodules and let $B$-grpmod denote the category of finitely-generated projective graded left $B$-supermodules. In both categories, we take the morphisms from $M$ to $N$ to be $\operatorname{Hom}_{B}(M, N)$. For each $n \in \mathbb{Z}$, we have a degree shift functor

$$
\{n\}: B \text {-grmod } \rightarrow B \text {-grmod, } M \mapsto M\{n\} .
$$

Here $M\{n\}$ is the same underlying vector space as $M$, with the same $B$-action, but a new grading given by $M\{n\}_{i, \epsilon}=M_{i-n, \epsilon}$. We have an analogous functor on the category of graded right $B$-supermodules, and hence on the category of graded $B$-superbimodules. We also have a parity shift functor

$$
\Pi: \text { B-grmod } \rightarrow B \text {-grmod, } \quad M \mapsto \Pi M,
$$

that switches the $\mathbb{Z}_{2}$-grading of the spaces, i.e. $(\Pi M)_{i, \epsilon}=M_{i, \epsilon+1}$. The action of $B$ on $\Pi M$ is given by $b \cdot m=(-1)^{\bar{b}} b m$, where $b m$ is the action on $M$. The parity shift functor $\Pi$ for a right supermodule switches the $\mathbb{Z}_{2}$-grading as above but, unlike the case of left supermodules, it does not change the signs in the action. For $n \in \mathbb{Z}$ and $\sigma \in \mathbb{Z}_{2}$, we define the functor

$$
\{n, \sigma\}: B \text {-grmod } \rightarrow \text { B-grmod, } M \mapsto M\{n, \sigma\}:=\Pi^{\sigma} M\{n\} .
$$

Both the degree shift and parity shift functors leave morphisms unchanged. Abusing notation, we will also sometimes use $\{n, \sigma\}$ to denote the map $M \rightarrow M\{n, \sigma\}$ that is the identity on elements of $M$.

For $M, N \in B$-grmod, we also define the $\left(\mathbb{Z} \times \mathbb{Z}_{2}\right)$-graded $\mathbb{F}$-vector space

$$
\operatorname{HOM}_{B}(M, N):=\bigoplus_{n \in \mathbb{Z}, \epsilon \in \mathbb{Z}_{2}} \operatorname{HOM}_{B}(M, N)_{n, \epsilon}, \quad \operatorname{HOM}_{B}(M, N)_{n, \epsilon}:=\operatorname{Hom}_{B}(M, N\{-n, \epsilon\}) .
$$

Note that we have an isomorphism of graded $\mathbb{F}$-vector spaces 


$$
\begin{aligned}
\operatorname{HOM}_{B}(M\{i, \epsilon\}, N\{j, \tau\}) & \cong \operatorname{HOM}_{B}(M, N)\{j-i, \tau+\epsilon\}, \\
f & \mapsto\{-i, \epsilon\} \circ f \circ\{i, \epsilon\} .
\end{aligned}
$$

and that, for $n \in \mathbb{Z}, \epsilon \in \mathbb{Z}_{2}$,

$$
\operatorname{HOM}_{B}(M, N)_{n, \epsilon}=\left\{f \in \operatorname{HOM}_{\mathbb{F}}(M, N)_{n, \epsilon} \mid f(b m)=(-1)^{\epsilon \bar{b}} b f(m) \forall b \in B, m \in M\right\} .
$$

If $M$ is a graded right $B$-supermodule, we sometimes will use the notation ${ }^{R} b(m)=m b$ for the operator of right multiplication. We will also need another family of operators involving a sign. For each homogeneous $b \in B$, we define an $\mathbb{F}$-linear operator

$$
{ }^{r} b: M \rightarrow M, \quad{ }^{r} b(m):=(-1)^{\bar{b} \bar{m}} m b \quad \text { for all homogeneous } m \in M \text {. }
$$

Notice that, if $\bar{b}=0$, then ${ }^{r} b={ }^{R} b$.

If $M$ is a graded $\left(B_{1}, B_{2}\right)$-superbimodule for graded superalgebras $B_{1}, B_{2}$, and $N$ is a graded $B_{1}$-supermodule, then $\operatorname{HOM}_{B_{1}}(M, N)$ is a graded left $B_{2}$-supermodule via the action

$$
b \cdot f=(-1)^{\bar{b} \bar{f}} f \circ{ }^{r} b, \quad b \in B_{2}, f \in \mathrm{HOM}_{B_{1}}(M, N),
$$

and $\operatorname{HOM}_{B_{1}}(N, M)$ is a graded right $B_{2}$-supermodule via the action

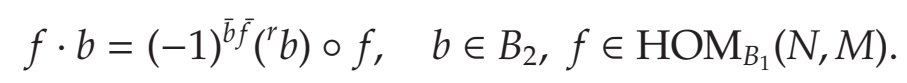

It is routine to verify that (3.2) is an isomorphism of graded left $B_{2}$-supermodules and graded right $B_{2}$-supermodules under the actions (3.4) and (3.5), respectively (when $M$ and $N$ have the appropriate structure).

If $M$ is a graded right $B_{2}$-supermodule, then it is also a graded $\left(\mathbb{F}, B_{2}\right)$-superbimodule and so the dual supermodule

$$
M^{\vee}:=\operatorname{HOM}_{\mathbb{F}}(M, \mathbb{F})
$$

is a graded left $B_{2}$-supermodule via the action (3.4). If $M$ is a graded left $B_{1}$-supermodule, then $M^{\vee}$ is a graded right $B_{1}$-supermodule via the action

$$
f \cdot b=f \circ{ }^{L} b, \quad f \in M^{\vee}, b \in B_{1} .
$$

Thus, if $M$ is a graded $\left(B_{1}, B_{2}\right)$-superbimodule, then $M^{\vee}$ is a graded $\left(B_{2}, B_{1}\right)$-superbimodule with action given, for homogeneous $b_{1} \in B_{1}, b_{2} \in B_{2}, f \in M^{\vee}$, by

$$
b_{2} \cdot f \cdot b_{1}=(-1)^{\bar{b}_{2} \bar{f}} f \circ{ }^{r} b_{2} \circ{ }^{L} b_{1}=(-1)^{\bar{b}_{2} \bar{f}+\bar{b}_{2} \bar{b}_{1}} f \circ{ }^{L} b_{1} \circ{ }^{r} b_{2} .
$$

Notice that, with this definition, $M^{\vee}$ is a $\left(B_{2}, B_{1}\right)$-bimodule since $\left(\left(b_{2} \cdot f\right) \cdot b_{1}\right)=\left(b_{2} \cdot\left(f \cdot b_{1}\right)\right)$. The difference in signs according to the order only appears when we want to express the formula in terms of the operators ${ }^{L} b_{1}$ and ${ }^{r} b_{2}$, which do not commute.

Suppose $M$ is a graded left $B$-supermodule, $N$ is a graded right $B$-supermodule, and $\tau$ is a graded superalgebra automorphism of $B$. Then we can define the twisted graded left $B$-supermodule ${ }^{\tau} M$ and twisted graded right $B$-supermodule $N^{\tau}$, with actions given by

$$
\begin{array}{cc}
b \cdot m \mapsto \tau(b) m, & b \in B, m \in M, \\
n \cdot b & \mapsto n \tau(b), \quad b \in B, n \in N,
\end{array}
$$

where juxtaposition denotes the original action of $B$ on $M$ and $N$. 
If $A$ and $B$ are graded superalgebras, then their tensor product $A \otimes B$ is also a graded superalgebra, with product

$$
\left(a_{1} \otimes b_{1}\right)\left(a_{2} \otimes b_{2}\right)=(-1)^{\bar{b}_{1} \bar{a}_{2}} a_{1} a_{2} \otimes b_{1} b_{2}, \quad a_{1}, a_{2} \in A, b_{1}, b_{2} \in B .
$$

Notice that there is an isomorphism $A \otimes B \cong B \otimes A$ of graded superalgebras defined by

$$
a \otimes b \mapsto(-1)^{\bar{a} \bar{b}} b \otimes a, \quad a \in A, b \in B .
$$

Given $M \in A$-grmod and $N \in B$-grmod, we define the outer tensor product $M \otimes N \in$ $(A \otimes B)$-grmod to be the vector space $M \otimes N$, with the action defined by

$$
(a \otimes b) \cdot(m \otimes n)=(-1)^{\bar{b} \bar{m}} a m \otimes b n, \quad a \in A, b \in B, m \in M, n \in N .
$$

The outer tensor product of morphisms is defined as follows. For $M_{1}, M_{2} \in A$-grmod, $N_{1}, N_{2} \in B$-grmod, $f \in \operatorname{HOM}_{A}\left(M_{1}, M_{2}\right)$, and $g \in \operatorname{HOM}_{B}\left(N_{1}, N_{2}\right)$, we define

$$
\begin{aligned}
f \otimes g & \in \mathrm{HOM}_{A \otimes B}\left(M_{1} \otimes N_{1}, M_{2} \otimes N_{2}\right), \\
(f \otimes g)(m \otimes n) & =(-1)^{\bar{g} \bar{m}} f(m) \otimes g(n), \quad m \in M_{1}, n \in N_{1} .
\end{aligned}
$$

We have an isomorphism of $\left(\mathbb{Z} \otimes \mathbb{Z}_{2}\right)$-graded $\mathbb{F}$-vector spaces

$$
\operatorname{HOM}_{A}\left(M_{1}, M_{2}\right) \otimes_{\mathbb{F}} \operatorname{HOM}_{B}\left(N_{1}, N_{2}\right) \cong \operatorname{HOM}_{A \otimes B}\left(M_{1} \otimes N_{1}, M_{2} \otimes N_{2}\right) \text {. }
$$

3.2. Grothendieck groups. Suppose that $B$ is a finite-dimensional graded superalgebra. We define the Grothendieck groups

$$
G_{0}^{\prime}(B)=\mathcal{K}_{0}(B \text {-grmod }) \text { and } K_{0}^{\prime}(B)=\mathcal{K}_{0}(B \text {-grpmod })
$$

Here $\mathcal{K}_{0}(B$-grmod) is defined to be the quotient of the free $\mathbb{Z}$-module with generators corresponding to finitely-generated graded $B$-supermodules, by the $\mathbb{Z}$-submodule generated by $M_{1}-M_{2}+M_{3}$ for all short exact sequences $0 \rightarrow M_{1} \rightarrow M_{2} \rightarrow M_{3} \rightarrow 0$ in $B$-grmod. In the same way, we define $\mathcal{K}_{0}(B$-grpmod), except we only consider projective objects (note that, in this case, we are in fact considering the split Grothendieck group, since all short exact sequences in $B$-grpmod split). If $C$ is a category, we denote the class of an object $M \in C$ in $\mathcal{K}_{0}(C)$ by $[M]$.

Let

$$
\mathbb{Z}_{q, \pi}:=\mathbb{Z}\left[q, q^{-1}, \pi\right] /\left(\pi^{2}-1\right) .
$$

We define actions of $\mathbb{Z}_{q, \pi}$ on $G_{0}^{\prime}(B)$ and $K_{0}^{\prime}(B)$ by setting

$$
\begin{array}{ccc}
\pi[M]=[\Pi M], & q^{n}[M]:=[M\{n\}], & \text { for all } M \in B \text {-grmod, } n \in \mathbb{Z}, \\
\pi[M]=[\Pi M], & q^{n}[M]:=[M\{-n\}], & \text { for all } M \in B \text {-grpmod, } n \in \mathbb{Z} .
\end{array}
$$

By (3.2), there is a natural $\mathbb{Z}_{q, \pi}$-bilinear map

$$
\langle-,-\rangle: K_{0}^{\prime}(B) \otimes G_{0}^{\prime}(B) \rightarrow \mathbb{Z}_{q, \pi}, \quad\langle[P],[M]\rangle=\operatorname{grdim}_{\mathbb{F}} \operatorname{HOM}_{B}(P, M),
$$

where, for a $\left(\mathbb{Z} \times \mathbb{Z}_{2}\right)$-graded vector space $V=\bigoplus_{n \in \mathbb{Z}, \epsilon \in \mathbb{Z}_{2}} V_{n, \epsilon}$ over $\mathbb{F}$, we define the graded dimension

$$
\operatorname{grdim}_{\mathbb{F}} V:=\sum_{n \in \mathbb{Z}, \epsilon \in \mathbb{Z}_{2}} q^{n} \pi^{\epsilon} \operatorname{dim}_{\mathbb{F}} V_{n, \epsilon} \in \mathbb{Z}_{q, \pi} .
$$

Note that, in some places in the literature, one defines $q^{n}[M]=[M\{n\}]$ for $M \in B$-grpmod, $n \in \mathbb{Z}$, so that the form (3.11) is $q$-sesquilinear. We choose a different convention because it simplifies certain arguments and notation to follow. 
Any simple graded $B$-supermodule is concentrated in one degree for the $\mathbb{Z}$-grading (recall that $B$ is $\mathbb{N}$-graded), and so, up to shift, it is isomorphic to an ungraded simple supermodule for the superalgebra $\tilde{B}_{0}:=B_{0,0} \oplus B_{0,1}$. Let $V_{1}, \ldots, V_{s}$ be a complete list of nonisomorphic simple $\tilde{B}_{0}$-supermodules. If $P_{i}$ is the projective cover, in the category $B$-grmod, of $V_{i}$ for $i=1, \ldots, s$, then $P_{1}, \ldots, P_{s}$ is a complete list of nonisomorphic indecomposable projective graded $B$-supermodules, up to shift.

If $\mathbb{F}$ is algebraically closed, then, for all $1 \leq i, j \leq s$,

$$
\left\langle\left[P_{i}\right],\left[V_{j}\right]\right\rangle= \begin{cases}\delta_{i j} & \text { if } V_{j} \text { is of type } \mathrm{M}, \\ (1+\pi) \delta_{i j} & \text { if } V_{j} \text { is of type Q. }\end{cases}
$$

(For this result and the definition of types $\mathrm{M}$ and $\mathrm{Q}$, see [Kle05, Lem. 12.2.3].) If $V_{1}, \ldots, V_{r}$ are irreducibles of type $\mathrm{M}$ and $V_{r+1}, \ldots, V_{s}$ are irreducibles of type $\mathrm{Q}$, we have isomorphisms of $\mathbb{Z}_{q, \pi}$-modules

$$
\begin{aligned}
& G_{0}^{\prime}(B) \cong \bigoplus_{i=1}^{r} \mathbb{Z}_{q, \pi}\left[V_{i}\right] \oplus \bigoplus_{j=r+1}^{s}\left(\mathbb{Z}_{q, \pi} /(\pi-1)\right)\left[V_{j}\right], \\
& K_{0}^{\prime}(B) \cong \bigoplus_{i=1}^{r} \mathbb{Z}_{q, \pi}\left[P_{i}\right] \oplus \bigoplus_{j=r+1}^{s}\left(\mathbb{Z}_{q, \pi} /(\pi-1)\right)\left[P_{j}\right] .
\end{aligned}
$$

Note that, if $B$ has graded left supermodules of type $Q$, then the bilinear form (3.11) is degenerate, since $1+\pi$ is a zero divisor. Furthermore, $K_{0}^{\prime}(B)$ and $G_{0}^{\prime}(B)$ are not free $\mathbb{Z}_{q, \pi}$-modules. We remedy this situation as follows. Let

$$
\mathbb{k}= \begin{cases}\mathbb{Z}_{q, \pi} & \text { if all simple graded left } B \text {-supermodules are of type } M, \\ \mathbb{Z}\left[\frac{1}{2}, q, q^{-1}\right] & \text { if } B \text { has a simple graded left supermodule of type } Q .\end{cases}
$$

We will identify $\mathbb{Z}\left[\frac{1}{2}, q, q^{-1}\right]$ with $\mathbb{Z}_{q, \pi}\left[\frac{1}{2}\right] /(\pi-1)$. Thus, $\mathbb{Z}\left[\frac{1}{2}, q, q^{-1}\right]$ is naturally a $\mathbb{Z}_{q, \pi}$-module and we have $\pi=1$ in $\mathbb{Z}\left[\frac{1}{2}, q, q^{-1}\right]$. Define

$$
G_{0}(B)=G_{0}^{\prime}(B) \otimes_{\mathbb{Z}_{q, \pi}} \mathbb{k}, \quad K_{0}(B)=K_{0}^{\prime}(B) \otimes_{\mathbb{Z}_{q, \pi}} \mathbb{k} .
$$

Then (3.11) induces a pairing

$$
\langle-,-\rangle: K_{0}(B) \otimes G_{0}(B) \rightarrow \mathbb{k} .
$$

If $\mathbb{F}$ is algebraically closed, we have

$$
G_{0}(B) \cong \bigoplus_{i=1}^{s} \mathbb{k}\left[V_{i}\right], \quad K_{0}(B) \cong \bigoplus_{i=1}^{s} \mathbb{k}\left[P_{i}\right]
$$

and the pairing (3.13) is perfect.

Suppose $\varphi: B \rightarrow A$ is a graded superalgebra homomorphism, possibly not preserving the identity element. In particular $\varphi \in \operatorname{HOM}_{\mathbb{F}}(B, A)_{0,0}$ because $\varphi\left(1_{B}\right) \in A_{0,0}$ is an idempotent. Then we can consider $A$ as a graded left $B$-supermodule via the action $b \cdot a=\varphi(b) a$. Similarly, we can consider $A$ as a right $B$-supermodule. Then we have induction and restriction functors

$$
\begin{gathered}
\operatorname{Ind}_{B}^{A}: B \text {-grmod } \rightarrow A \text {-grmod, } \quad \operatorname{Res}_{B}^{A}: A \text {-grmod } \rightarrow B \text {-grmod, } \\
\operatorname{Ind}_{B}^{A} N:=A \varphi\left(1_{B}\right) \otimes_{B} N, \quad N \in B \text {-grmod, } \\
\operatorname{Res}_{B}^{A} M:=\operatorname{HOM}_{A}\left(A \varphi\left(1_{B}\right), M\right) \cong{ }_{B} \varphi\left(1_{B}\right) A \otimes_{A} M, \quad M \in A \text {-grmod, }
\end{gathered}
$$

where ${ }_{B} \varphi\left(1_{B}\right) A$ denotes $\varphi\left(1_{B}\right) A$ considered as a $(B, A)$-superbimodule and the left $B$-action on $\operatorname{HOM}_{A}\left(A \varphi\left(1_{B}\right), M\right)$ is given by using the right action of $B$ on $A$, as in (3.4). The 
isomorphism above is given by the map $f \mapsto \varphi\left(1_{B}\right) \otimes f\left(\varphi\left(1_{B}\right)\right)$ for $f \in \operatorname{HOM}_{A}\left(A \varphi\left(1_{B}\right), M\right)$. This isomorphism is natural in $M$, and so we have an isomorphism of functors $\operatorname{Res}_{B}^{A} \cong$ ${ }_{B} \varphi\left(1_{B}\right) A \otimes_{A}-$. It follows from the standard tensor-hom adjunction that $\operatorname{Ind}_{B}^{A}$ is left adjoint to $\operatorname{Res}_{B}^{A}$.

\section{TOWERS OF GRADED SUPERALGEBRAS}

In this section, we define towers of graded superalgebras and various properties they may possess. These towers will be the main ingredient in our categorification. Recall that $\mathbb{F}$ is an arbitrary field of characteristic not equal to two.

Definition 4.1 (Tower of graded superalgebras). Let $\Lambda \cong \mathbb{N}^{r}$ be as in the beginning of Section 2, and let $A=\bigoplus_{\lambda \in \Lambda} A_{\lambda}$ be a $\Lambda$-graded superalgebra over $\mathbb{F}$ with multiplication $\rho: A \otimes A \rightarrow A$. Define

$\mathbb{k}= \begin{cases}\mathbb{Z}_{q, \pi} & \text { if, for all } \lambda \in \Lambda, \text { all simple graded left } A_{\lambda} \text {-supermodules are of type } \mathrm{M}, \\ \mathbb{Z}\left[\frac{1}{2}, q, q^{-1}\right] & \text { if, for some } \lambda \in \Lambda, A_{\lambda} \text { has a simple graded left supermodule of type } \mathrm{Q} .\end{cases}$

Then $A$ is called a tower of graded superalgebras if the following conditions are satisfied.

TA1: Each graded piece $A_{\lambda}, \lambda \in \Lambda$, is a finite-dimensional $\mathbb{N}$-graded superalgebra as in Section 3.1 (with a different multiplication than that of $A$ ) with a unit $1_{\lambda}$. We have $A_{0} \cong \mathbb{F}$.

TA2: The external multiplication $\rho_{m, n}: A_{\lambda} \otimes A_{\mu} \rightarrow A_{\lambda+\mu}$ is a homomorphism of graded superalgebras for all $\lambda, \mu \in \Lambda$ (possibly not sending $1_{\lambda} \otimes 1_{\mu}$ to $1_{\lambda+\mu}$ ).

TA3: We have that $A_{\lambda+\mu}$ is a two-sided projective $\left(A_{\lambda} \otimes A_{\mu}\right)$-supermodule with the action defined by

$$
a \cdot(b \otimes c)=a \rho_{\lambda, \mu}(b \otimes c) \quad \text { and } \quad(b \otimes c) \cdot a=\rho_{\lambda, \mu}(b \otimes c) a,
$$

for all $\lambda, \mu \in \Lambda, a \in A_{\lambda+\mu}, b \in A_{\lambda}, c \in A_{\mu}$.

TA4: For each $\lambda \in \Lambda$, the pairing (3.13), with $B=A_{\lambda}$, is perfect. (Note that this condition is automatically satisfied if $\mathbb{F}$ is algebraically closed.)

For the remainder of this section we assume that $A$ is a tower of graded superalgebras. Let

$$
\mathcal{G}(A)=\bigoplus_{\lambda \in \Lambda} G_{0}\left(A_{\lambda}\right) \text { and } \mathcal{K}(A)=\bigoplus_{\lambda \in \Lambda} K_{0}\left(A_{\lambda}\right) .
$$

Then we have a perfect pairing $\langle-,-\rangle: \mathcal{K}(A) \times \mathcal{G}(A) \rightarrow \mathbb{k}$ given by

$$
\langle[P],[M]\rangle= \begin{cases}\operatorname{grdim}_{\mathbb{F}} \operatorname{HOM}_{A_{\lambda}}(P, M) & \text { if } P \in A_{\lambda} \text {-grpmod and } M \in A_{\lambda} \text {-grmod } \\ & \text { for some } \lambda \in \Lambda, \\ 0 & \text { otherwise. }\end{cases}
$$

We also define a perfect pairing $\langle-,-\rangle:(\mathcal{K}(A) \otimes \mathcal{K}(A)) \times(\mathcal{G}(A) \otimes \mathcal{G}(A)) \rightarrow \mathbb{k}$ by $\langle[P] \otimes[Q],[M] \otimes[N]\rangle=\left\{\begin{array}{c}\operatorname{grdim}_{\mathbb{F}}\left(\mathrm{HOM}_{A_{\lambda} \otimes A_{\mu}}(P \otimes Q, M \otimes N)\right) \quad \text { if } P \in A_{\lambda} \text {-grpmod, } Q \in A_{\mu} \text {-grpmod } \\ \text { and } M \in A_{\lambda} \text {-grmod, } N \in A_{\mu} \text {-grmod for some } \lambda, \mu \in \Lambda, \\ 0 \quad \text { otherwise. }\end{array}\right.$ 
It follows from (3.10) that, for all $P, Q, M, N$,

$$
\langle[P] \otimes[Q],[M] \otimes[N]\rangle=\langle[P],[M]\rangle\langle[Q],[N]\rangle .
$$

Consider the direct sums of categories

$$
A \text {-grmod := } \bigoplus_{\lambda \in \Lambda} A_{\lambda} \text {-grmod, } A \text {-grpmod }:=\bigoplus_{\lambda \in \Lambda} A_{\lambda} \text {-grpmod. }
$$

For $r \in \mathbb{N}_{+}$, we define

$$
\begin{aligned}
A-\operatorname{grmod}^{\otimes r} & :=\bigoplus_{\lambda_{1}, \ldots, \lambda_{r} \in \Lambda}\left(A_{\lambda_{1}} \otimes \cdots \otimes A_{\lambda_{r}}\right) \text {-grmod, } \\
A-\operatorname{grpmod}^{\otimes r} & :=\bigoplus_{\lambda_{1}, \ldots, \lambda_{r} \in \Lambda}\left(A_{\lambda_{1}} \otimes \cdots \otimes A_{\lambda_{r}}\right) \text {-grpmod. }
\end{aligned}
$$

Then, for $i, j \in\{1, \ldots, r\}, i<j$, we define $S_{i j}: A-\operatorname{grmod}^{\otimes r} \rightarrow A-\operatorname{grmod}^{\otimes r}$ to be the endofunctor that interchanges the $i$ th and $j$ th factors, that is, the endofunctor arising from the isomorphism

$$
A_{\lambda_{1}} \otimes \cdots \otimes A_{\lambda_{r}} \cong A_{\lambda_{1}} \otimes \cdots \otimes A_{\lambda_{i-1}} \otimes A_{\lambda_{j}} \otimes A_{\lambda_{i+1}} \otimes \cdots \otimes A_{\lambda_{j-1}} \otimes A_{\lambda_{i}} \otimes A_{\lambda_{j+1}} \otimes \cdots \otimes A_{\lambda_{r}}
$$

that maps the element $a_{1} \otimes \cdots \otimes a_{r}$ to

$$
(-1)^{\left(\bar{a}_{i}+\bar{a}_{j}\right)\left(\bar{a}_{i+1}+\cdots+\bar{a}_{j-1}\right)+\bar{a}_{i} \bar{a}_{j}} a_{1} \otimes \cdots \otimes a_{i-1} \otimes a_{j} \otimes a_{i+1} \otimes \cdots \otimes a_{j-1} \otimes a_{i} \otimes a_{j+1} \otimes \cdots \otimes a_{r} .
$$

We use the same notation to denote the analogous endofunctor on $A$-grpmod ${ }^{\otimes r}$.

We also have the following functors:

$$
\begin{array}{r}
\nabla: A \text {-grmod }{ }^{\otimes 2} \rightarrow A \text {-grmod, }\left.\quad \nabla\right|_{\left(A_{\lambda} \otimes A_{\mu}\right) \text {-grmod }}=\operatorname{Ind}_{A_{\lambda} \otimes A_{\mu}{ }^{\prime}}^{A_{\lambda+\mu}} \\
\Delta: A \text {-grmod } \rightarrow A \text {-grmod }{ }^{\otimes 2},\left.\quad \Delta\right|_{A_{\lambda} \text {-grmod }}=\bigoplus_{\mu+v=\lambda} \operatorname{Res}_{A_{\mu} \otimes A_{\nu}{ }^{\prime}}^{A_{\lambda}} \\
\eta: \text { grVect } \rightarrow A \text {-grmod, } \quad \eta(V)=V \in A_{0} \text {-grmod for } V \in \text { grVect, } \\
\varepsilon: A \text {-grmod } \rightarrow \text { grVect, } \quad \varepsilon(V)= \begin{cases}V & \text { if } V \in A_{0} \text {-grmod, } \\
0 & \text { otherwise. }\end{cases}
\end{array}
$$

In the above, we have identified $A_{0}$-grmod with the category grVect of finite-dimensional $\left(\mathbb{Z} \times \mathbb{Z}_{2}\right)$-graded vector spaces over $\mathbb{F}$. Replacing $A$-grmod by $A$-grpmod above, we also have the functors $\nabla, \Delta, \eta$ and $\varepsilon$ on $A$-grpmod. Since the above functors are all exact (we use axiom TA3 here), they induce a multiplication, comultiplication, unit and counit on $\mathcal{G}(A)$ and $\mathcal{K}(A)$. We use the same notation to denote these induced maps.

Since induction is left adjoint to restriction, $\nabla$ is left adjoint to $\Delta$. However, there are examples of towers of graded superalgebras for which induction is not right adjoint to restriction (see, for example, Section 8). Nevertheless, we often have something quite close to this property. Any graded superalgebra automorphism $\psi_{\lambda}$ of $A_{\lambda}$ induces an isomorphism of categories $\Psi_{\lambda}: A_{\lambda}$-grmod $\rightarrow A_{\lambda}$-grmod (which restricts to an isomorphism of categories $\Psi_{\lambda}: A_{\lambda}$-grpmod $\rightarrow A_{\lambda}$-grpmod) by twisting the $A_{\lambda}$-action as in (3.8). Then $\Psi:=\bigoplus_{\lambda \in \Lambda} \Psi_{\lambda}$ is an automorphism of the categories $A$-grmod and $A$-grpmod. It induces automorphisms, which we also denote by $\Psi$, of $\mathcal{G}(A)$ and $\mathcal{K}(A)$. Similarly, if $\delta=\left(\delta_{\lambda}\right)_{\lambda \in \Lambda} \in \mathbb{Z}^{\Lambda}$, and $\sigma=\left(\sigma_{\lambda}\right)_{\lambda \in \Lambda} \in \mathbb{Z}_{2}^{\Lambda}$, then $\{\delta, \sigma\}=\bigoplus_{\lambda}\left\{\delta_{\lambda}, \sigma_{\lambda}\right\}$ defines a grading shift automorphism of the categories $A$-grmod and $A$-grpmod. 
Definition 4.2 (Conjugate shifted adjointness). Suppose that, for $\lambda \in \Lambda, \psi_{\lambda}$ is an automorphism of graded superalgebras $A_{\lambda} \rightarrow A_{\lambda}, \delta_{\lambda} \in \mathbb{Z}$, and $\sigma_{\lambda} \in \mathbb{Z}_{2}$, and define $\Psi$ and $\{\delta, \sigma\}$ as above. Then, given a tower of graded superalgebras $A$, we say that induction is conjugate shifted right adjoint to restriction (and restriction is conjugate shifted left adjoint to induction) with conjugation $\Psi$ and shift $\{\delta, \sigma\}$ if $\nabla$ is right adjoint to $\Psi^{\otimes 2}\left\{\delta^{\otimes 2}, \sigma^{\otimes 2}\right\} \Delta \Psi^{-1}\{-\delta,-\sigma\}$.

There are some situations in which conjugate shifted adjointness is automatically satisfied. For example, in Section 6, we will see that this is the case for towers of Frobenius graded superalgebras.

Definition 4.3 (Strong tower of graded superalgebras). Suppose $\chi=\left(\chi^{\prime}, \chi^{\prime \prime}\right)$ is a pair of biadditive maps $\chi^{\prime}, \chi^{\prime \prime}: \Lambda \times \Lambda \rightarrow \mathbb{Z}, d \in \mathbb{Z}_{,} \in \in \mathbb{Z}_{2}, \delta=\left(\delta_{\lambda}\right)_{\lambda \in \Lambda} \in \mathbb{Z}^{\Lambda}$, and $\sigma=\left(\sigma_{\lambda}\right)_{\lambda \in \Lambda} \in \mathbb{Z}_{2}^{\Lambda}$. We say that a tower of graded superalgebras $A$ is strong with twist $(\chi, d, \epsilon)$, conjugation $\Psi$ and shift $(\delta, \sigma)$ if the following two conditions are satisfied:

S1: Induction is conjugate shifted right adjoint to restriction with conjugation $\Psi$ and shift $\{\delta, \sigma\}$, and there exists a biadditive map $\kappa: \Lambda \times \Lambda \rightarrow \mathbb{Z}$ such that the maps

$$
\kappa_{\delta}, \kappa_{\sigma}: \Lambda \times \Lambda \rightarrow \mathbb{Z}, \quad \kappa_{\alpha}(\lambda, \mu)=\alpha_{\lambda+\mu}-\alpha_{\lambda}-\alpha_{\mu} \quad \text { for } \alpha=\delta, \sigma,
$$

are of the form $\kappa_{\delta}=d \kappa, \kappa_{\sigma}=\epsilon \kappa$.

S2: We have an isomorphism of functors

$$
\Delta \nabla \cong \nabla^{\otimes 2} S_{23}\{\chi\} \Delta^{\otimes 2},
$$

where $\{\chi\}$ is the endofunctor of $A$-grmod ${ }^{\otimes 4}$ given on $\left(A_{\lambda_{1}} \otimes A_{\lambda_{2}} \otimes A_{\lambda_{3}} \otimes A_{\lambda_{4}}\right)$-grmod by $\{\chi\}\left(M_{1} \otimes M_{2} \otimes M_{3} \otimes M_{4}\right)=\left(M_{1} \otimes M_{2} \otimes M_{3} \otimes M_{4}\right)\left\{d \chi^{\prime}\left(\lambda_{2}, \lambda_{3}\right)+d \chi^{\prime \prime}\left(\lambda_{1}, \lambda_{4}\right), \epsilon \chi^{\prime}\left(\lambda_{2}, \lambda_{3}\right)+\right.$ $\left.\epsilon \chi^{\prime \prime}\left(\lambda_{1}, \lambda_{4}\right)\right\}$.

We say that the tower has trivial twist if $\chi=0$ or $d=\epsilon=0$.

Definition 4.4 (Dualizing and compatible towers of graded superalgebras). We say that a tower of graded superalgebras $A$ is dualizing if, under the operations $(4.4), \mathcal{K}(A)$ and $\mathcal{G}(A)$ are twisted Hopf algebras which are twisted dual, in the sense of Definition 2.2, under the bilinear form (4.2) (i.e. the perfect pairing (4.2) is a twisted Hopf pairing). In addition, we say that $A$ is compatible if the dual pair $(\mathcal{K}(A), \mathcal{G}(A))$ is compatible, as in Definition 2.4.

To simplify terminology, from now on we will just use the terms 'strong tower', 'dualizing tower' and 'compatible tower', and will consider implied the specifier 'of graded superalgebras'. In the case that $\Lambda=\mathbb{N}$ and each $A_{n}$ lies in degree $(0,0)$, the following result was proved in [SY15, Prop. 3.7].

Proposition 4.5. Suppose $A$ is a strong tower with conjugation $\Psi$, twist $(\chi, d, \epsilon)$, and shift $\{\delta, \sigma\}$. Then the following statements are equivalent.

(a) The tower A is dualizing with $a\left(q^{d} \pi^{\epsilon}, 0, \kappa\right)$-twisted Hopfpairing, where $\kappa$ is as in Definition 4.3.

(b) We have $\Psi^{\otimes 2} \Delta \Psi^{-1}(P) \cong \Delta(P)$ for all $P \in A$-grpmod.

(c) We have $\Psi^{\otimes 2} \Delta \Psi^{-1}=\Delta$ as maps $\mathcal{K}(A) \rightarrow \mathcal{K}(A) \otimes \mathcal{K}(A)$.

In particular, $A$ is dualizing if $\Psi=\mathrm{Id}$ or, more generally, if $\Psi$ acts trivially on $\mathcal{K}(A)$. 
Proof. First assume that (b) holds. The proof of the associativity of $\nabla$, the coassociativity of $\Delta$, and the first and third equations in Definition 2.1 are almost identical to the arguments found in the proof of [BL09, Th. 3.6]. It then follows immediately from axiom S2 in Definition 4.3 that $\mathcal{G}(A)$ is a $\left(q^{d} \pi^{\epsilon}, \chi\right)$-Hopf algebra and that $\mathcal{K}(A)$ is a $\left(q^{d} \pi^{\epsilon},-\chi\right)$-Hopf algebra, where $-\chi=\left(-\chi^{\prime},-\chi^{\prime \prime}\right)$. It remains to prove that

$$
\langle[P], \nabla([M] \otimes[N])\rangle=\left(q^{d} \pi^{\epsilon}\right)^{\kappa(|M|,|N|)}\langle\Delta([P]),[M] \otimes[N]\rangle,
$$

for all $M \in A_{\lambda}$-grmod, $N \in A_{\mu}$-grmod, $P \in A_{\lambda+\mu}$-grpmod. However, under our assumptions, we have

$$
\begin{aligned}
\operatorname{Hom}_{A_{\lambda+\mu}}(P, \nabla(M \otimes N)) & \cong \operatorname{Hom}_{A_{\lambda} \otimes A_{\mu}}\left(\Psi^{\otimes 2}\left\{\delta^{\otimes 2}, \sigma^{\otimes 2}\right\} \Delta \Psi^{-1}\{-\delta,-\sigma\}(P), M \otimes N\right) \\
& \cong \operatorname{Hom}_{A_{\lambda} \otimes A_{\mu}}\left(\Psi^{\otimes 2} \Delta \Psi^{-1}(P)\left\{\delta_{\lambda}+\delta_{\mu}-\delta_{\lambda+\mu}, \sigma_{\lambda}+\sigma_{\mu}-\sigma_{\lambda+\mu}\right\}, M \otimes N\right) \\
& \cong \operatorname{Hom}_{A_{\lambda} \otimes A_{\mu}}(\Delta(P), M \otimes N)\{d \kappa(\lambda, \mu), \epsilon \kappa(\lambda, \mu)\},
\end{aligned}
$$

which immediately implies (4.6). Thus (a) is true.

Now suppose (a) is true. Then, for all $P \in A_{\lambda+\mu}$ grpmod, $M \in A_{\lambda}$-grmod and $N \in$ $A_{\mu}$-grmod we have

$$
\left\langle\Psi^{\otimes 2} \Delta \Psi^{-1}([P]),[M] \otimes[N]\right\rangle=\left(q^{d} \pi^{\epsilon}\right)^{-\kappa(\lambda, \mu)}\langle[P], \nabla([M] \otimes[N])\rangle=\langle\Delta([P]),[M] \otimes[N]\rangle,
$$

where the first equality holds by the assumption that induction is conjugate shifted right adjoint to restriction and the second equality holds by (a). Then (c) follows from the nondegeneracy of the bilinear form.

The fact that (b) and (c) are equivalent follows from the fact that every short exact sequence of projective supermodules splits. Thus, for $P, Q \in A$-grpmod, we have $[P]=[Q]$ in $\mathcal{K}(A)$ if and only if $P \cong Q$.

\section{Categorification of the twisted Heisenberg double and its Fock space}

In this section we apply the constructions of Section 2 to the dual pair $(G(A), \mathcal{K}(A))$ arising from a compatible tower $A$. We will see that some natural subalgebras of the twisted Heisenberg double arise in this situation. We then prove our main result (Theorem 5.7), the categorification of the Fock space representation of the twisted Heisenberg double. As a corollary, we have a categorification of the twisted Heisenberg double itself (see Corollary 5.8). We fix a compatible tower $A$ (although many of the results of this section only require that $A$ be dualizing).

Definition $5.1\left(\mathfrak{h}(A), \mathcal{F}(A), \mathcal{G}_{\text {proj }}(A)\right)$. We define

$$
\mathfrak{h}(A)=\mathfrak{h}(\mathcal{G}(A), \mathcal{K}(A)) \text { and } \mathcal{F}(A)=\mathcal{F}(\mathcal{G}(A), \mathcal{K}(A)) .
$$

For each $\lambda \in \Lambda, A_{\lambda}$-grpmod is a full subcategory of $A_{\lambda}$-grmod. The inclusion functor induces the Cartan map $\mathcal{K}(A) \rightarrow \mathcal{G}(A)$, which is antilinear in the sense that it is linear in $\mathbb{Z}$ and $\pi$, but interchanges $q$ and $q^{-1}$. Let $\mathcal{G}_{\text {proj }}(A)$ denote the image of the Cartan map.

Let

$$
H^{-}=\mathcal{K}(A), H^{+}=\mathcal{G}(A), H_{\text {proj }}^{+}=\mathcal{G}_{\text {proj }}(A), \mathfrak{h}=\mathfrak{h}(A), \mathcal{F}=\mathcal{F}(A) .
$$

To avoid confusion between $\mathcal{G}(A)$ and $\mathcal{K}(A)$, we will write $[M]_{+}$to denote the class of a finitely-generated (possibly projective) graded $A_{\lambda}$-supermodule in $H^{+}$and $[M]_{-}$to 
denote the class of a finitely-generated graded projective $A_{\lambda}$-supermodule in $H^{-}$. If $P \in$ $A_{v}$-grpmod and $N \in\left(A_{\lambda-v} \otimes A_{v}\right)$-grmod, then we have a natural graded $A_{\lambda-v}$-supermodule structure on $\mathrm{HOM}_{A_{v}}(P, N)$ given by

$$
a \cdot f={ }^{L}(a \otimes 1) \circ f, \quad a \in A_{\lambda-v}, f \in \operatorname{Hom}_{A_{v}}(P, N) .
$$

Lemma 5.2. If $\lambda, v \in \Lambda, P \in A_{v}$-grpmod and $N \in A_{\lambda}$-grmod, then we have

$$
[P]_{-} \cdot[N]_{+}= \begin{cases}0 & \text { if } v \notin \lambda, \\ {\left[\operatorname{HOM}_{A_{v}}\left(P, \operatorname{Res}_{A_{\lambda-v} \otimes A_{v}}^{A_{\lambda}} N\right)\right]_{+}} & \text {if } v \leq \lambda,\end{cases}
$$

where $v \leq \lambda$ if and only if there exists $\mu \in \Lambda$ such that $v+\mu=\lambda$. Here $\cdot$ denotes the action of $\mathfrak{h}$ on $\mathcal{F}$ and $\operatorname{HOM}_{A_{v}}\left(P, \operatorname{Res}_{A_{\lambda-v} \otimes A_{v}}^{A_{\lambda}} N\right)$ is viewed as an $A_{\lambda-v}$-supermodule as in (5.2).

Proof. The proof is analogous to that of [SY15, Lem. 3.10].

Proposition 5.3. We have that $\mathrm{H}_{\text {proj }}^{+}$is a subalgebra of $\mathrm{H}^{+}$that is invariant under the left regular action of $\mathrm{H}^{-}$.

Proof. The proof is analogous to that of [SY15, Prop. 3.12].

Definition 5.4 (The projective twisted Heisenberg double $\mathfrak{h}_{\text {proj }}(A)$ ). By Proposition 5.3, $\mathfrak{h}_{\text {proj }}=\mathfrak{h}_{\text {proj }}(A):=H_{\text {proj }}^{+} \# H^{-}$is a subalgebra of $\mathfrak{h}$. In other words, $\mathfrak{h}_{\text {proj }}$ is the subalgebra of $\mathfrak{h}$ generated by $H_{\text {proj }}^{+}$and $H^{-}$(viewing the latter two as $\mathbb{k}$-submodules of $\mathfrak{h}$ as in Definition 2.5). We call $\mathfrak{h}_{\text {proj }}$ the projective twisted Heisenberg double associated to $A$.

Definition 5.5 (Fock space $\mathcal{F}_{\text {proj }}(A)$ of $\left.\mathfrak{h}_{\text {proj }}\right)$. By Proposition 5.3 , the algebra $\mathfrak{h}_{\text {proj }}$ acts on $H_{\text {proj. }}^{+}$We call this the lowest weight Fock space representation of $\mathfrak{h}_{\text {proj }}$ and denote it by $\mathcal{F}_{\text {proj }}=\mathcal{F}_{\text {proj }}(A)$. Note that this representation is generated by the lowest weight vacuum vector $1 \in H_{\text {proj }}^{+}$.

Recall the direct sums of categories

$$
A \text {-grmod }=\bigoplus_{\lambda \in \Lambda} A_{\lambda} \text {-grmod, } A \text {-grpmod }=\bigoplus_{\lambda \in \Lambda} A_{\lambda} \text {-grpmod }
$$

For each $M \in A_{\mu}$-grmod, $\mu \in \Lambda$, define the functor $\operatorname{Ind}_{M}: A$-grmod $\rightarrow A$-grmod by

$$
\operatorname{Ind}_{M}(N)=\operatorname{Ind}_{A_{\mu} \otimes A_{\lambda}}^{A_{\mu+\lambda}}(M \otimes N) \in A_{\mu+\lambda} \text {-grmod, } \quad N \in A_{\lambda} \text {-mod, } \lambda \in \Lambda \text {. }
$$

For each $P \in A_{v}$-grpmod, $v \in \Lambda$, define the functor $\operatorname{Res}_{P}: A$-grmod $\rightarrow A$-grmod by

$$
\operatorname{Res}_{P}(N)=\operatorname{HOM}_{A_{v}}\left(P, \operatorname{Res}_{A_{\lambda-v} \otimes A_{v}}^{A_{\lambda}} N\right) \in A_{\lambda-v} \text {-grmod, } N \in A_{\lambda} \text {-grmod, } \lambda \in \Lambda,
$$

where $\operatorname{Res}_{P}(N)$ is interpreted to be the zero object of $A$-grmod if $\lambda \nsucceq \nu$. Since $\operatorname{Ind}_{P}(A$-grpmod $) \subseteq A$-grpmod, $\operatorname{Res}_{P}(A$-grpmod $) \subseteq A$-grpmod, for all $P \in A$-grpmod, we have the induced functors $\operatorname{Ind}_{P}, \operatorname{Res}_{P}: A$-grpmod $\rightarrow A$-grpmod for $P \in A$-grpmod.

Because the functors Ind $\operatorname{In}_{M}$ and $\operatorname{Res}_{P}$ are exact for all $M \in A$-grmod and $P \in A$-grpmod, they induce endomorphisms $\left[\operatorname{Ind}_{M}\right]$ and $\left[\operatorname{Res}_{P}\right]$ of $\mathcal{G}(A)$. Similarly, Ind ${ }_{P}$ and $\operatorname{Res}_{P}$ induce endomorphisms $\left[\operatorname{Ind}_{P}\right]$ and $\left[\operatorname{Res}_{P}\right]$ of $\mathcal{G}_{\text {proj }}(A)$ for all $P \in A$-grpmod.

Proposition 5.6. Suppose A is a compatible tower. 
(a) For all $M, N \in A$-grmod and $P \in A$-grpmod, we have

$$
([M] \#[P])([N])=\left[\operatorname{Ind}_{M}\right] \circ\left[\operatorname{Res}_{P}\right]([N])=\left[\operatorname{Ind}_{M} \circ \operatorname{Res}_{P}(N)\right] \in \mathcal{G}(A) .
$$

(b) For all $Q, P, R \in A$-grpmod, we have

$$
([Q] \#[P])([R])=\left[\operatorname{Ind}_{Q}\right] \circ\left[\operatorname{Res}_{P}\right]([R])=\left[\operatorname{Ind}_{Q} \circ \operatorname{Res}_{P}(R)\right] \in \mathcal{G}_{\text {proj }}(A) .
$$

Proof. This follows from the definition of the multiplication in $\mathcal{G}(A)$ and Lemma 5.2.

Part (a) (resp. part (b)) of Proposition 5.6 shows how the action of $\mathfrak{h}$ on $\mathcal{F}$ (resp. $\mathfrak{h}_{\text {proj }}$ on $\mathcal{F}_{\text {proj }}$ ) is induced by functors on $\bigoplus_{\lambda \in \Lambda} A_{\lambda}$-grmod (resp. $\bigoplus_{\lambda \in \Lambda} A_{\lambda}$-grpmod). Typically a categorification of a representation consists of isomorphisms of such functors which lift the algebra relations. As we now describe, this can be done if the tower is also strong. For the remainder of this section, we fix a strong compatible tower $A$ with twist $(\chi, d, \epsilon)$, shift $(\delta, \sigma)$ and biadditive map $\kappa$ as in Definition 4.3.

Since the natural action of $\mathfrak{h}$ on $H^{+}$is faithful by [RS, Th. 4.3(d)], the algebra structure on $\mathfrak{h}$ is uniquely determined by the fact that $H^{ \pm}$are subalgebras and by (2.5), which gives the commutation relation between $H^{+}$and $H^{-}$. Now, recalling that $\left(\gamma^{\prime}, \gamma^{\prime \prime}\right)=(0, \kappa),(2.5)$ is equivalent to the following equalities in End $\mathrm{H}^{+}$:

$$
{ }^{R} x^{*} \circ{ }^{L} a=\nabla\left(\Delta(x)^{\dagger}(a \otimes-)\right), \quad x \in H^{-}, a \in H^{+},
$$

where, for homogeneous $x, y \in H^{-}$, we define the operator $(x \otimes y)^{\dagger} \in \operatorname{End}\left(H^{+} \otimes H^{+}\right)$by

$$
(x \otimes y)^{\dagger}(a \otimes b)=\left(q^{d} \pi^{\epsilon}\right)^{\kappa(|x|,|y|)+\chi^{\prime}(|x|,|b|-|y|)+\chi^{\prime \prime}(|a|-|x|,|y|){ }^{R} x^{*}(a) \otimes{ }^{R} y^{*}(b),}
$$

and extend by linearity. For $Q \in\left(A_{v} \otimes A_{\rho}\right)$-grpmod, $M \in A_{\mu}$-grmod, and $N \in A_{\lambda}$-grmod, define

$$
\begin{aligned}
\operatorname{Res}_{Q}^{\dagger}(M \otimes N):=\operatorname{HOM}_{A_{\nu} \otimes A_{\rho}}\left(Q, S_{23}\left(\operatorname{Res}_{A_{\mu-\nu} \otimes A_{v}}^{A_{\mu}} M \otimes \operatorname{Res}_{A_{\lambda-\rho} \otimes A_{\rho}}^{A_{\lambda}} N\right)\right) \\
\left\{d \kappa(v, \rho)+d \chi^{\prime}(v, \lambda-\rho)+d \chi^{\prime \prime}(\mu-v, \rho), \epsilon \kappa(v, \rho)+\epsilon \chi^{\prime}(v, \lambda-\rho)+\epsilon \chi^{\prime \prime}(\mu-v, \rho)\right\} .
\end{aligned}
$$

Theorem 5.7. Suppose that $A$ is a strong compatible tower. Then we have the following isomorphisms of functors for all $M, N \in A$-grmod and $P, Q \in A$-grpmod.

$$
\begin{gathered}
\operatorname{Ind}_{M} \circ \operatorname{Ind}_{N} \cong \operatorname{Ind}_{\nabla(M \otimes N)}, \\
\operatorname{Res}_{P} \circ \operatorname{Res}_{Q} \cong \operatorname{Res}_{\nabla(P \otimes Q),} \\
\operatorname{Res}_{P} \circ \operatorname{Ind}_{M} \cong \nabla \operatorname{Res}_{\Delta(P)}^{+}(M \otimes-) .
\end{gathered}
$$

In particular, the above yields a categorification of the lowest weight Fock space representations of $\mathfrak{h}(A)$ and $\mathfrak{h}_{\text {proj }}(A)$.

Proof. The proofs of (5.4) and (5.5) are identical to the proofs of the analogous statements in [SY15, Th. 3.18] and are therefore omitted. It remains to prove (5.6). For simplicity, in what follows we will use the notation $\operatorname{Res}_{\sigma, v}^{\sigma+v}$ for $\operatorname{Res}_{A_{\sigma} \otimes A_{v}}^{A_{\sigma+v}}$ and an analogous notation for induction functors. We will also use a similar notation if the restriction or induction involves any number of tensor factors. We have, for $P \in A_{\nu}$-grpmod, $M \in A_{\mu}$-grmod, and $L \in A_{\rho}$-grmod,

$\operatorname{Res}_{P} \circ \operatorname{Ind}_{M}(L)=\operatorname{HOM}_{A_{v}}\left(P, \operatorname{Res}_{\mu+\rho-v, v}^{\mu+\rho} \operatorname{Ind}_{\mu, \rho}^{\mu+\rho}(M \otimes L)\right)$ 


$$
\begin{aligned}
& \cong \operatorname{HOM}_{A_{v}}\left(P, \bigoplus_{\alpha+\beta=\nu} \operatorname{Ind}_{\mu-\alpha, \rho-\beta, \alpha, \beta}^{\mu+\rho-v, v} S_{23} \operatorname{Res}_{\mu-\alpha, \alpha, \rho-\beta, \beta}^{\mu, \rho}(M \otimes L)\right)\left\{\zeta^{\prime}, \zeta^{\prime \prime}\right\} \\
& \cong \operatorname{HOM}_{A_{v}}\left(P, \bigoplus_{\alpha+\beta=v} \operatorname{Ind}_{\mu-\alpha, \rho-\beta, \nu}^{\mu+\rho-v, v} \operatorname{Ind}_{\mu-\alpha, \rho-\beta, \alpha, \beta}^{\mu-\alpha, \rho-\beta, v} S_{23} \operatorname{Res}_{\mu-\alpha, \alpha, \rho-\beta, \beta}^{\mu, \rho}(M \otimes L)\right)\left\{\zeta^{\prime}, \zeta^{\prime \prime}\right\} \\
& \cong \bigoplus_{\alpha+\beta=v} \operatorname{Ind}_{\mu-\alpha, \rho-\beta}^{\mu+\rho-v} \operatorname{HOM}_{A_{v}}\left(P, \operatorname{Ind}_{\mu-\alpha, \rho-\beta, \alpha, \beta}^{\mu-\alpha, \rho-\beta, v} S_{23} \operatorname{Res}_{\mu-\alpha, \alpha, \rho-\beta, \beta}^{\mu, \rho}(M \otimes L)\right)\left\{\zeta^{\prime}, \zeta^{\prime \prime}\right\} \\
& \cong \underset{\alpha+\beta=v}{ } \operatorname{Ind}_{\mu-\alpha, \rho-\beta}^{\mu+\rho-v} \operatorname{HOM}_{A_{\alpha} \otimes A_{\beta}}\left(\operatorname{Res}_{\alpha, \beta}^{v}(P)\left\{-\kappa_{\delta}(\alpha, \beta),-\kappa_{\sigma}(\alpha, \beta)\right\}, S_{23} \operatorname{Res}_{\mu-\alpha, \alpha, \rho-\beta, \beta}^{\mu, \rho}(M \otimes L)\right)\left\{\zeta^{\prime}, \zeta^{\prime \prime}\right\} \\
& \cong \underset{\alpha+\beta=v}{\bigoplus_{\alpha n d}^{\mu+\rho-v}} \operatorname{IOM}_{\mu-\alpha, \rho-\beta} \operatorname{HOM}_{A_{\alpha} \otimes A_{\beta}}\left(\operatorname{Res}_{\alpha, \beta}^{v}(P)\{-d \kappa(\alpha, \beta),-\epsilon \kappa(\alpha, \beta)\}, S_{23} \operatorname{Res}_{\mu-\alpha, \alpha, \rho-\beta, \beta}^{\mu, \rho}(M \otimes L)\right)\left\{\zeta^{\prime}, \zeta^{\prime \prime}\right\} \\
& \cong \nabla \operatorname{Res}_{\Delta(P)}^{+}(M \otimes L),
\end{aligned}
$$

where

$$
\zeta^{\prime}=d \chi^{\prime}(\alpha, \rho-\beta)+d \chi^{\prime \prime}(\mu-\alpha, \beta), \quad \zeta^{\prime \prime}=\epsilon \chi^{\prime}(\alpha, \rho-\beta)+\epsilon \chi^{\prime \prime}(\mu-\alpha, \beta) .
$$

The first isomorphism above follows from (4.5). In the fourth isomorphism, we use the fact that induction is conjugate shifted right adjoint to restriction and that the tower is dualizing. Since all of the above isomorphisms are natural in $L$, this proves (5.6).

The final assertion of the theorem follows as explained in the paragraph preceding the statement of the theorem. In particular, the isomorphisms (5.4) and (5.5) categorify the multiplication in $\mathcal{G}(A)$ and $\mathcal{K}(A)$, respectively, and the isomorphism (5.6) categorifies the relation (5.3).

Corollary 5.8. Let $\mathcal{H}$ be the full subcategory of End(A-grmod) generated by $\operatorname{Ind}_{M}, M \in$ $A$-grmod, and $\operatorname{Res}_{P}, P \in A$-grpmod, under composition and degree shift. Then $\mathcal{K}_{0}(\mathcal{H}) \cong \mathfrak{h}(A)$.

Proof. By Theorem 5.7, we have a surjective map

$$
\mathfrak{h}(A) \rightarrow \mathcal{K}_{0}(\mathcal{H}), \quad[M] \#[P] \mapsto\left[\operatorname{Ind}_{M}\right] \circ\left[\operatorname{Res}_{P}\right], \quad M \in A \text {-grmod, } P \in A \text {-grpmod }
$$

By [RS, Th. 4.3(a)], the kernel of this map must be zero, hence the map is also injective.

Note that the categorifications of Theorem 5.7 and Corollary 5.8 do not rely on a particular presentation of the twisted Heisenberg double $\mathfrak{h}(A)$.

\section{Frobenius graded superalgebras}

Many existing constructions in categorification involve Frobenius (super)algebras. In this section, we show that towers of Frobenius graded superalgebras automatically satisfy some of the axioms of a strong tower. In particular, induction is always conjugate shifted right adjoint to restriction (see Proposition 6.7). Our definition of a Frobenius superalgebra is more general that the definition typically appearing in the literature (those definitions require $\sigma=0$ in Definition 6.1 below). In addition, we consider the graded version of these algebras.

Definition 6.1 (Frobenius graded superalgebra). Suppose $\delta \in \mathbb{Z}$ and $\sigma \in \mathbb{Z}_{2}$. We say that a finite-dimensional graded superalgebra $B$ is a Frobenius graded superalgebra of degree $(-\delta, \sigma)$ if one of the following three equivalent conditions holds: 
(a) There is an isomorphism (homogeneous of degree zero) of graded left $B$-supermodules

$$
\varphi: B \rightarrow B^{\vee}\{\delta, \sigma\}=\operatorname{HOM}_{\mathbb{F}}(B, \mathbb{F})\{\delta, \sigma\}=\operatorname{HOM}_{\mathbb{F}}(B, \mathbb{F}\{\delta, \sigma\}) .
$$

Here $B^{\vee}$ is a graded left $B$-supermodule as in (3.4).

(b) There exists a nondegenerate invariant graded $\mathbb{F}$-bilinear form $(-,-): B \times B \rightarrow$ $\mathbb{F}\{\delta, \sigma\}$. By invariant, we mean that

$$
(a b, c)=(a, b c) \quad \text { for all } a, b, c \in B .
$$

By graded, we mean that $\left(B_{\lambda, \epsilon}, B_{\lambda^{\prime}, \epsilon^{\prime}}\right) \subseteq \mathbb{F}\{\delta, \sigma\}_{\lambda+\lambda^{\prime}, \epsilon+\epsilon^{\prime}}$. In other words, $B_{\lambda, \epsilon} \perp B_{\lambda^{\prime}, \epsilon^{\prime}}$ unless $\lambda+\lambda^{\prime}=\delta$ and $\epsilon+\epsilon^{\prime}=\sigma$, since we view $\mathbb{F}$ as lying in degree zero.

(c) There exists an $\mathbb{F}$-linear graded (i.e. homogeneous of degree zero) map tr: $B \rightarrow$ $\mathbb{F}\{\delta, \sigma\}$, called the trace map, such that the kernel of tr contains no nonzero left ideals of $B$.

The relationship between the various structures in Definition 6.1 is as follows: For $a, b \in B$, we have

$$
\varphi(b)(a)=(-1)^{\bar{a} \bar{b}}(a, b), \quad \operatorname{tr}(b)=(b, 1)=(1, b), \quad(a, b)=\operatorname{tr}(a b) .
$$

The proof that the conditions in Definition 6.1 are equivalent is a straightforward generalization of the analogous fact for Frobenius algebras.

Remark 6.2. Note that the choice of $\delta$ in Definition 6.1 is unique, since it is the maximal value of $\delta$ such that $B_{\delta, \epsilon} \neq 0$ for some $\epsilon \in \mathbb{Z}_{2}$. However, the $\mathbb{Z}_{2}$-degree ( $\sigma$ in Definition 6.1) of $B$ is not necessarily unique. In what follows, when we say that $B$ is a Frobenius graded superalgebra of degree $(-\delta, \sigma)$, we assume that we have fixed a choice of trace map (equivalently, isomorphism $\varphi$ or bilinear form) satisfying the conditions in Definition 6.1 for that degree.

Lemma 6.3 (Nakayama automorphism). If $B$ is a Frobenius graded superalgebra, then there exists an automorphism $\psi: B \rightarrow B$ of graded superalgebras such that $(a, b)=(-1)^{\bar{a} \bar{b}}(b, \psi(a))$ for all homogeneous $a, b \in B$. This automorphism is called the Nakayama automorphism of $B$.

The following proof is a rather straightforward generalization of the proof of the existence of the Nakayama automorphism in the non-graded, non-super setting (see, for example, [Lam99, §16E]).

Proof. For a fixed $a \in B$, the map $b \mapsto(-1)^{\bar{a} \bar{b}}(a, b)$ is an $\mathbb{F}$-linear functional on $B$. Thus, it is of the form $b \mapsto(b, \psi(a))$ for some $\psi(a) \in B$. It is straightforward to verify that the map $\psi: B \rightarrow B$ thus defined is an automorphism of $\mathbb{F}$-vector spaces. Now, suppose $B$ is of degree $(-\delta, \sigma)$. For $\lambda, \lambda^{\prime} \in \mathbb{Z}$ and $\epsilon, \epsilon^{\prime} \in \mathbb{Z}_{2}$, we have

$$
\left(B_{\lambda^{\prime}, \epsilon^{\prime}}, \psi\left(B_{\lambda, \epsilon}\right)\right)=\left(B_{\lambda, \epsilon}, B_{\lambda^{\prime}, \epsilon^{\prime}}\right)=0 \quad \text { if } \lambda+\lambda^{\prime} \neq \delta \text { or } \epsilon+\epsilon^{\prime} \neq \sigma .
$$

Thus $\psi\left(B_{\lambda, \epsilon}\right) \subseteq B_{\lambda, \epsilon}$, and so $\psi$ is a map of $\left(\mathbb{Z}, \mathbb{Z}_{2}\right)$-graded vector spaces. Finally, for all homogeneous $a, b, c \in B$, we have

$$
\begin{aligned}
(b, \psi(a c))=(-1)^{\bar{a} \bar{b}+\bar{c} \bar{b}}(a c, b)=(-1)^{\bar{a} \bar{b}+\bar{c} \bar{b}}(a, c b) & =(-1)^{\bar{c} \bar{b}+\bar{a} \bar{c}}(c b, \psi(a)) \\
& =(-1)^{\bar{c} \bar{b}+\bar{a} \bar{c}}(c, b \psi(a))=(b \psi(a), \psi(c))=(b, \psi(a) \psi(c)) .
\end{aligned}
$$


Hence $\psi(a c)=\psi(a) \psi(c)$ by the nondegeneracy of the bilinear form, and so $\psi$ is an automorphism of graded superalgebras.

Lemma 6.4. For $i=1,2$, let $B_{i}$ be a Frobenius graded superalgebra of degree $\left(-\delta_{i}, \sigma_{i}\right)$, with trace map $\operatorname{tr}_{i}$ and Nakayama automorphism $\psi_{i}$. Then $B_{1} \otimes B_{2}$ is a Frobenius graded superalgebra of degree $\left(-\delta_{1}-\delta_{2}, \sigma_{1}+\sigma_{2}\right)$, with trace map $\operatorname{tr}_{1} \otimes \operatorname{tr}_{2}$ and Nakayama automorphism $\psi_{1} \otimes \psi_{2}$. The invariant bilinear form on $B_{1} \otimes B_{2}$ is defined in terms of the invariant bilinear forms on $B_{1}$ and $B_{2}$ by

$$
\left(b_{1} \otimes b_{2}, c_{1} \otimes c_{2}\right):=(-1)^{\bar{b}_{2} \bar{c}_{1}}\left(b_{1}, c_{1}\right)\left(b_{2}, c_{2}\right),
$$

for all homogeneous $b_{1}, c_{1} \in B_{1}, b_{2}, c_{2} \in B_{2}$.

Proof. The straightforward proof of this lemma is left to the reader.

Lemma 6.5. If $B$ is a Frobenius graded superalgebra of degree $(-\delta, \sigma)$, then we have an isomorphism of graded $(B, B)$-superbimodules

$$
B^{\psi} \cong B^{\vee}\{\delta, \sigma\} .
$$

Proof. By Definition 6.1(a) we have an isomorphism $\varphi: B \rightarrow B^{\vee}\{\delta, \sigma\}$ of graded left $B$ supermodules. It remains to show that, under the isomorphism $\varphi$, the right $B$-action on $B^{\vee}\{\delta, \sigma\}$ defined by (3.6) corresponds to the right $B$-action on $B$ twisted by the Nakayama automorphism. For homogeneous $a, b, c \in B$, we have

$$
\begin{aligned}
(\varphi(b) \cdot a)(c)=\left(\varphi(b) \circ{ }^{L} a\right)(c)=\varphi(b)(a c)=(-1)^{\bar{a} \bar{b}+\bar{b} \bar{c}}(a c, b) & =(-1)^{\bar{a} \bar{b}+\bar{b} \bar{c}}(a, c b) \\
=(-1)^{\overline{\bar{a}}+\bar{b} \bar{c}}(c b, \psi(a)) & =(-1)^{\overline{\bar{c}}+\bar{b} \bar{c}}(c, b \psi(a))=\varphi(b \psi(a))(c) .
\end{aligned}
$$

Thus, $\varphi(b) \cdot a=\varphi(b \psi(a))$.

Suppose $\tau$ is an automorphism of $B$. Then, if $M$ is a graded left (resp. right) $B$ supermodule, it is straightforward to verify that

$$
\left({ }^{\tau} M\right)^{\vee} \cong\left(M^{\vee}\right)^{\tau} \quad\left(\text { resp. }\left(M^{\tau}\right)^{\vee} \cong{ }^{\tau}\left(M^{\vee}\right)\right)
$$

as right (resp. left) graded $B$-supermodules. Note also that, if $M$ is a graded left $B$ supermodule and $N$ is a graded right $B$-supermodule, then we have an isomorphism

$$
N^{\tau} \otimes_{B} M \cong N \otimes_{B}{ }^{\tau^{-1}} M .
$$

Lemma 6.6. For $i=1,2$, let $B_{i}$ be a Frobenius graded superalgebra of degree $\left(-\delta_{i}, \sigma_{i}\right)$, with Nakayama automorphism $\psi_{i}$. Let $M$ be a finite-dimensional graded $\left(B_{1}, B_{2}\right)$-superbimodule that is projective as a left $B_{1}$-supermodule and also as a right $B_{2}$-supermodule. Then the functor

$$
M \otimes_{B_{2}}-: B_{2} \text {-grmod } \rightarrow B_{1} \text {-grmod }
$$

has a right adjoint functor

$$
\left(M^{\vee}\right)^{\psi_{1}^{-1}}\left\{\delta_{1}, \sigma_{1}\right\} \otimes_{B_{1}}-: B_{1} \text {-grmod } \rightarrow B_{2} \text {-grmod, }
$$

and a left adjoint functor

$$
\psi_{2}\left(M^{\vee}\right)\left\{\delta_{2}, \sigma_{2}\right\} \otimes_{B_{1}}-: B_{1} \text {-grmod } \rightarrow B_{2} \text {-grmod. }
$$


Proof. Recall the left $B_{2}$-action and right $B_{1}$-action on $\operatorname{HOM}_{B_{1}}\left(M, B_{1}\right)$ defined by (3.4) and (3.5), respectively. Suppose $X$ is a graded left $B_{1}$-supermodule. Consider the natural $\mathbb{F}$-linear map

$$
\Phi: \operatorname{HOM}_{B_{1}}\left(M, B_{1}\right) \otimes_{B_{1}} X \rightarrow \operatorname{HOM}_{B_{1}}(M, X), \quad \alpha \otimes x \mapsto\left(m \mapsto(-1)^{\bar{x} \bar{m}} \alpha(m) x\right) .
$$

If $M \cong B_{1}$ as a left $B_{1}$-supermodule, then $\Phi$ is an isomorphism between the graded vector spaces $\mathrm{HOM}_{B_{1}}\left(B_{1}, B_{1}\right) \otimes_{B_{1}} X$ and $\operatorname{HOM}_{B_{1}}\left(B_{1}, X\right)$, which are both naturally isomorphic to $X$. If $M$ is free as a left $B_{1}$-supermodule, then we also get an isomorphism in a similar way. It follows from additivity that $\Phi$ is an isomorphism whenever $M$ is a finite-dimensional projective graded $B_{1}$-supermodule. The isomorphism $\Phi$ is natural in $M$, hence it is also an isomorphism of graded left $B_{2}$-supermodules, for the action (3.4), when $M$ satisfies the conditions in the statement of the lemma. Since $\Phi$ is also natural in $X$, we have an isomorphism of functors

$$
\operatorname{HOM}_{B_{1}}\left(M, B_{1}\right) \otimes_{B_{1}}-\cong \operatorname{HOM}_{B_{1}}(M,-)
$$

from $B_{1}$-grmod to $B_{2}$-grmod.

From Lemma 6.5 , we have an isomorphism of graded right $\left(B_{1}, B_{1}\right)$-superbimodules $B_{1} \cong \operatorname{HOM}_{\mathbb{F}}\left(B_{1}, \mathbb{F}\right)^{\psi_{1}^{-1}}\left\{\delta_{1}, \sigma_{1}\right\}$. We then get isomorphisms of graded $\left(B_{2}, B_{1}\right)$-superbimodules

$$
\begin{aligned}
\operatorname{HOM}_{B_{1}}\left(M, B_{1}\right) & \cong \operatorname{HOM}_{B_{1}}\left(M, \operatorname{HOM}_{\mathbb{F}}\left(B_{1}, \mathbb{F}\right)^{\psi_{1}^{-1}}\left\{\delta_{1}, \sigma_{1}\right\}\right) \\
& \cong \operatorname{HOM}_{B_{1}}\left(M, \operatorname{HOM}_{\mathbb{F}}\left(B_{1}, \mathbb{F}\right)\right)^{\psi_{1}^{-1}}\left\{\delta_{1}, \sigma_{1}\right\} \\
& \cong \operatorname{HOM}_{\mathbb{F}}\left(B_{1} \otimes_{B_{1}} M, \mathbb{F}\right)^{\psi_{1}^{-1}}\left\{\delta_{1}, \sigma_{1}\right\} \\
& \cong \operatorname{HOM}_{\mathbb{F}}(M, \mathbb{F})_{1}^{\psi_{1}^{-1}}\left\{\delta_{1}, \sigma_{1}\right\} \\
& \cong\left(M^{\vee}\right)^{\psi_{1}^{-1}}\left\{\delta_{1}, \sigma_{1}\right\},
\end{aligned}
$$

where the third isomorphism follows from the tensor-hom adjunction (it is a straightforward exercise to verify that this is an isomorphism of superbimodules). It follows that we have isomorphisms of functors

$$
\left(M^{\vee}\right)^{\psi_{1}^{-1}}\left\{\delta_{1}, \sigma_{1}\right\} \otimes_{B_{1}}-\cong \operatorname{HOM}_{B_{1}}\left(M, B_{1}\right) \otimes_{B_{1}}-\cong \operatorname{HOM}_{B_{1}}(M,-) .
$$

Since the functor $M \otimes_{B_{2}}$ - has the right adjoint $\operatorname{HOM}_{B_{1}}(M,-)$, the first statement of the lemma holds.

For the second statement, remark that if $M$ is finite dimensional and projective as a left $B_{1}$-supermodule and as a right $B_{2}$-supermodule, then $M^{\vee}$ is also finite dimensional and projective as a left $B_{2}$-supermodule and right $B_{1}$-supermodule, and thus so is $\psi_{2}\left(M^{\vee}\right)$. Thus we can apply the first part of the proof to conclude that $\psi_{2} M^{\vee}\left\{\delta_{2}, \sigma_{2}\right\} \otimes_{B_{1}}$ - is left adjoint to

$$
\left(\left({ }^{\psi_{2}} M^{\vee}\left\{\delta_{2}, \sigma_{2}\right\}\right)^{\vee}\right)^{\psi_{2}^{-1}}\left\{\delta_{2}, \sigma_{2}\right\} \otimes_{B_{2}}-\cong\left(\left(\psi_{2} M^{\vee}\right)^{\vee}\right)^{\psi_{2}^{-1}} \otimes_{B_{2}}-.
$$

But, using (6.1), we have isomorphisms of $\left(B_{1}, B_{2}\right)$-superbimodules

$$
\left(\left({ }^{\psi_{2}} M^{\vee}\right)^{\vee}\right)^{\psi_{2}^{-1}} \cong\left(\left(\left(M^{\vee}\right)^{\vee}\right)^{\psi_{2}}\right)^{\psi_{2}^{-1}} \cong\left(M^{\vee}\right)^{\vee} \cong M,
$$

which concludes the proof. 
Proposition 6.7. If $A$ is a tower of algebras such that each $A_{\lambda}, \lambda \in \Lambda$, is a Frobenius graded superalgebra of degree $\left(-\delta_{\lambda}, \sigma_{\lambda}\right)$ with Nakayama automorphism $\psi_{\lambda}$, then induction is conjugate shifted right adjoint to restriction with conjugation $\Psi$ and shifting $\{\delta, \sigma\}$ (in the notation of Definition 4.2).

Proof. We apply Lemma 6.6 with $B_{1}=A_{\lambda+\mu}, B_{2}=A_{\lambda} \otimes A_{\mu}$, and $M=A_{\lambda+\mu}$, considered as an $\left(A_{\lambda+\mu}, A_{\lambda} \otimes A_{\mu}\right)$-superbimodule in the natural way. In the notation of Lemma 6.6, we have

$$
\psi_{2}=\psi_{\lambda} \otimes \psi_{\mu}, \quad \delta_{2}=\delta_{\lambda}+\delta_{\mu}, \quad \sigma_{2}=\sigma_{\lambda}+\sigma_{\mu} .
$$

By Lemma 6.5, we have

$$
\left(A_{\lambda+\mu}\right)^{\vee} \cong\left(A_{\lambda+\mu}\right)^{\psi_{\lambda+\mu}}\left\{-\delta_{\lambda+\mu},-\sigma_{\lambda+\mu}\right\}
$$

as $\left(A_{\lambda+\mu}, A_{\lambda+\mu}\right)$-superbimodules. Restricting the action on the left, we have

$$
M^{\vee} \cong{ }_{A_{\lambda} \otimes A_{\mu}}\left(A_{\lambda+\mu}\right)^{\psi_{\lambda+\mu}}\left\{-\delta_{\lambda+\mu},-\sigma_{\lambda+\mu}\right\},
$$

as $\left(A_{\lambda} \otimes A_{\mu}, A_{\lambda+\mu}\right)$-superbimodules. Then, by Lemma 6.6, the left adjoint to Ind $\operatorname{In}_{A_{\lambda} \otimes A_{\mu}}^{A_{\lambda}}=$ $M \otimes_{B_{2}}-$ is

$$
\begin{aligned}
\psi_{\lambda} \otimes \psi_{\mu}\left(A_{\lambda} \otimes A_{\mu}\right. & \left.\left(A_{\lambda+\mu}\right)\right)^{\psi_{\lambda+\mu}}\left\{\delta_{\lambda}+\delta_{\mu}-\delta_{\lambda+\mu}, \sigma_{\lambda}+\sigma_{\mu}-\sigma_{\lambda+\mu}\right\} \otimes_{A_{\lambda+\mu}}- \\
& =\left(\Psi_{\lambda} \otimes \Psi_{\mu}\right) \operatorname{Res}_{A_{\lambda} \otimes A_{\mu}}^{A_{\lambda+\mu}} \Psi_{\lambda+\mu}^{-1}\left\{\delta_{\lambda}+\delta_{\mu}-\delta_{\lambda+\mu}, \sigma_{\lambda}+\sigma_{\mu}-\sigma_{\lambda+\mu}\right\},
\end{aligned}
$$

where the exponent of -1 on $\Psi_{\lambda+\mu}$ comes from (6.2).

\section{TOWERS OF WREATH PRODUCT ALGEBRAS}

In this section, we introduce a large class of examples of strong compatible towers. This class includes several towers that have been considered in the literature (see Example 7.1).

Suppose that $B$ is a graded superalgebra over an algebraically closed field $\mathbb{F}$. Recall that $B^{\otimes n}$ is a graded superalgebra with multiplication

$$
\left(b_{1} \otimes \cdots \otimes b_{n}\right)\left(b_{1}^{\prime} \otimes \cdots \otimes b_{n}^{\prime}\right)=(-1)^{\sum_{i<j} b_{i} \bar{b}_{j}^{\prime}} b_{1} b_{1}^{\prime} \otimes \cdots \otimes b_{n} b_{n}^{\prime}, \quad b_{i}, b_{i}^{\prime} \in B, 1 \leq i \leq n .
$$

Then $S_{n}$ acts on $B^{\otimes n}$ by superpermutations. More precisely, if $s_{k} \in S_{n}$ is the simple transposition $(k, k+1)$ for $1 \leq k \leq n-1$, then

$$
s_{k} \cdot\left(b_{1} \otimes \cdots \otimes b_{n}\right)=(-1)^{\bar{b}_{k} \bar{b}_{k+1}} b_{1} \otimes \cdots \otimes b_{k-1} \otimes b_{k+1} \otimes b_{k} \otimes b_{k+2} \otimes \cdots \otimes b_{n} .
$$

(Note that we consider the action by superpermutations since the action by usual permutations does not, in general, give an action of $S_{n}$ on $B^{\otimes n}$ by superalgebra automorphisms.) We can thus form the algebra $A_{n}:=B^{\otimes n} \rtimes S_{n}$. As an F-vector space, we have $B^{\otimes n} \rtimes S_{n} \cong B^{\otimes n} \otimes \mathbb{F}\left[S_{n}\right]$. This inherits a $\left(\mathbb{Z} \times \mathbb{Z}_{2}\right)$-grading from $B$ by declaring $S_{n}$ to be in degree zero. The multiplication is determined by the fact that $B^{\otimes n}$ and $\mathbb{k}\left[S_{n}\right]$ are sub-superalgebras and

$$
\tau \beta \tau^{-1}=\tau \cdot \beta, \quad \beta \in B^{\otimes n}, \tau \in S_{n} .
$$

Motivated by the analogous construction for groups, we call $B^{\otimes n} \rtimes S_{n}$ a wreath product algebra. 
Example 7.1. If $B$ is the rank one Clifford superalgebra, then $B^{\otimes n} \rtimes S_{n}$ is isomorphic to the Sergeev superalgebra (see [Kle05, Lem. 13.2.3]). Thus, the tower of Sergeev superalgebras fits into the framework of the current section. The algebras considered in [CL12] are also of the form presented here.

We define an external multiplication on $A=\bigoplus_{n \in \mathbb{N}} A_{n}$ by

$$
\begin{aligned}
\rho_{m, n}: A_{m} \otimes A_{n} \cong\left(B^{\otimes m} \rtimes S_{m}\right) \otimes\left(B^{\otimes n} \rtimes\right. & \left.S_{n}\right) \\
& \cong B^{\otimes(m+n)} \rtimes\left(S_{m} \times S_{n}\right) \hookrightarrow B^{\otimes(m+n)} \rtimes S_{m+n} \cong A_{m+n},
\end{aligned}
$$

induced by the natural inclusion $S_{m} \times S_{n} \hookrightarrow S_{m+n}$. Axioms TA1 and TA2 follow immediately.

Recall that, as a left $\left(\mathbb{F}\left[S_{m}\right] \otimes \mathbb{F}\left[S_{n}\right]\right)$-module, $\mathbb{F}\left[S_{m+n}\right]$ has a basis given by minimal length representatives of the cosets $\left(S_{m} \times S_{n}\right) \backslash S_{m+n}$. It follows that, as a left $\left(A_{m} \otimes A_{n}\right)$-supermodule, we have

$$
A_{m+n}=\bigoplus_{w \in X_{m, n}}\left(A_{m} \otimes A_{n}\right) w,
$$

where $X_{m, n}$ is a set of minimal length representatives of the cosets $\left(S_{m} \times S_{n}\right) \backslash S_{m+n}$. Thus $A_{m+n}$ is a projective left $\left(A_{m} \otimes A_{n}\right)$-supermodule. Similarly, it is also a projective right $\left(A_{m} \otimes A_{n}\right)$-supermodule and so axiom TA3 is satisfied. Finally, TA4 is satisfied since $\mathbb{F}$ is algebraically closed.

Now suppose that $B$ is a Frobenius graded superalgebra of degree $(-\delta, \sigma)$. If $\operatorname{tr}_{B}: B \rightarrow$ $\mathbb{F}\{\delta, \sigma\}$ is the trace map of $B$, then, by Lemma $6.4, B^{\otimes n}$ is a Frobenius graded superalgebra with trace map $\operatorname{tr}_{B}^{\otimes n}$. Recall also that $\mathbb{F}\left[S_{n}\right]$ is a Frobenius graded superalgebra (concentrated in degree zero) with trace map $\operatorname{tr}_{S_{n}}: \mathbb{F}\left[S_{n}\right] \rightarrow \mathbb{F}$ given by $\operatorname{tr}_{S_{n}}(w)=\delta_{w, w_{0}}$, where $w_{0}$ is the longest element of $S_{n}$.

Lemma 7.2. The algebra $A_{n}$ is a Frobenius graded superalgebra of degree $(-n \delta, n \sigma)$, with trace map $\operatorname{tr}_{n}: A_{n} \rightarrow \mathbb{F}$ given by $\operatorname{tr}_{n}=\operatorname{tr}_{B}^{\otimes n} \otimes \operatorname{tr}_{S_{n}}$. The corresponding Nakayama automorphism $\psi_{n}: A_{n} \rightarrow A_{n}$ is given by

$$
\begin{gathered}
\psi_{n}\left(b_{1} \otimes \cdots \otimes b_{n}\right)=(-1)^{\sum_{i<j} \bar{b}_{i} \bar{b}_{j}} \psi_{B}\left(b_{n}\right) \otimes \cdots \otimes \psi_{B}\left(b_{1}\right), \quad b_{1}, \ldots, b_{n} \in B, \\
\psi_{n}\left(s_{i}\right)=(-1)^{\sigma} s_{n-i}, \quad i=1, \ldots, n-1,
\end{gathered}
$$

where $\psi_{B}: B \rightarrow B$ is the Nakayama automorphism of $B$.

Proof. To show that $A_{n}$ is a Frobenius graded superalgebra with trace map $\operatorname{tr}_{n}$, it suffices to show that ker $\operatorname{tr}_{n}$ contains no nonzero left ideals. Let $I$ be a nonzero left ideal of $A_{n}$. Then choose a nonzero element $a=\sum_{w \in S_{n}} a_{w} w \in I$. Without loss of generality, we may assume that $a_{w_{0}} \neq 0$ (otherwise consider $w_{0} \tau^{-1} a$, where $\tau$ is a maximal length element of the set $\left.\left\{w \in S_{n} \mid a_{w} \neq 0\right\}\right)$. Now, since $B^{\otimes n}$ is a Frobenius algebra, the ideal of $B^{\otimes n}$ generated by $a_{w_{0}}$ is not contained in $\operatorname{ker}_{B} \operatorname{tr}_{B}^{\otimes n}$. Thus, there exists $c \in B^{\otimes n}$ such that $\operatorname{tr}_{B}^{\otimes n}\left(c a_{w_{0}}\right) \neq 0$. Then $\operatorname{tr}_{n}(c a) \neq 0$. Thus, $I$ is not contained in $\operatorname{tr}_{n}$. It is clear that the degree of the Frobenius graded superalgebra $A_{n}$ is $(-n \delta, n \sigma)$.

Now suppose $\beta \in B^{\otimes n}$ and $\tau \in S_{n}$ such that $\tau s_{i}=w_{0}$ (hence $s_{n-i} \tau=w_{0}$ ). Write $\beta=\beta^{\prime}+\beta^{\prime \prime}$, where

$$
\beta^{\prime} \in\left(\bar{B}_{\sigma}\right)^{\otimes n}, \quad \beta^{\prime \prime} \in \bigoplus_{\substack{\epsilon_{1}, \ldots, \epsilon_{n} \\ \text { not all equal to } \sigma}}\left(\bar{B}_{\epsilon_{1}} \otimes \cdots \otimes \bar{B}_{\epsilon_{n}}\right) .
$$


Then

$$
\begin{array}{r}
\operatorname{tr}_{n}\left((\beta \otimes \tau) s_{i}\right)=\operatorname{tr}_{n}\left(\beta \otimes w_{0}\right)=\operatorname{tr}_{B}^{\otimes n}\left(\beta^{\prime}\right)=(-1)^{\sigma} \operatorname{tr}_{B}^{\otimes n}\left(s_{n-i} \cdot \beta^{\prime}\right) \\
=(-1)^{\sigma} \operatorname{tr}_{B}^{\otimes n}\left(s_{n-i} \cdot \beta\right)=(-1)^{\sigma} \operatorname{tr}_{n}\left(s_{n-i}(\beta \otimes \tau)\right) .
\end{array}
$$

On the other hand, if $\tau s_{i} \neq w_{0}$ (in which case $s_{n-i} \tau \neq w_{0}$ ), then

$$
\operatorname{tr}_{n}\left((\beta \otimes \tau) s_{i}\right)=0=\operatorname{tr}_{n}\left(s_{n-i}(\beta \otimes \tau)\right) \text {. }
$$

Thus, $\psi_{n}\left(s_{i}\right)=(-1)^{\sigma} s_{n-i}$.

Finally, for homogeneous $b_{1}, \ldots, b_{n} \in B$ and $\beta \in B^{\otimes n}$, we have

$$
\begin{aligned}
\operatorname{tr}_{n}\left(\left(b_{1} \otimes \cdots \otimes b_{n}\right)\left(\beta \otimes w_{0}\right)\right) & =\operatorname{tr}\left(\left(b_{1} \otimes \cdots \otimes b_{n}\right) \beta \otimes w_{0}\right) \\
& =\operatorname{tr}_{B}^{\otimes n}\left(\left(b_{1} \otimes \cdots \otimes b_{n}\right) \beta\right) \\
& =(-1)^{|\beta|\left(\left|b_{1}\right|+\cdots+\left|b_{n}\right|\right)} \operatorname{tr}_{B}^{\otimes n}\left(\beta\left(\psi_{B}\left(b_{1}\right) \otimes \cdots \otimes \psi_{B}\left(b_{n}\right)\right)\right) \\
& =(-1)^{|\beta|\left(\left|b_{1}\right|+\cdots+\left|b_{n}\right|\right)}(-1)^{\sum_{i<j} \bar{b}_{i} \bar{b}_{j}} \operatorname{tr}_{n}\left(\left(\beta \otimes w_{0}\right)\left(\psi_{B}\left(b_{n}\right) \otimes \cdots \otimes \psi_{B}\left(b_{1}\right)\right)\right) .
\end{aligned}
$$

On the other hand, if $\tau \neq w_{0}$, then we have

$$
\operatorname{tr}_{n}\left(\left(b_{1} \otimes \cdots \otimes b_{n}\right)(\beta \otimes \tau)\right)=0=\operatorname{tr}_{n}\left((\beta \otimes \tau)\left(\psi_{B}\left(b_{n}\right) \otimes \cdots \otimes \psi_{B}\left(b_{1}\right)\right)\right) .
$$

This completes the proof.

Proposition 7.3. The tower $A$ is strong with trivial twist and conjugation $\Psi$ given by the Nakayama automorphism (see Proposition 6.7).

Proof. It follows from Lemma 7.2 and Proposition 6.7 that induction is conjugate shifted right adjoint to restriction. Since $\alpha_{n+m}-\alpha_{n}-\alpha_{n}=(n+m) \alpha-n \alpha-m \alpha=0$ for $\alpha=\delta, \sigma$, condition S1 of Definition 4.3 is satisfied with $d=\epsilon=0$ and $\kappa=0$.

To check S2, we formulate the isomorphism (4.5) in terms of superbimodules. Fix $n, m, k, \ell$ such that $n+m=k+\ell$ and set $K=n+m$. Let ${ }_{(k, \ell)}\left(A_{K}\right)_{(n, m)}$ denote $A_{K}$, thought of as a $\left(A_{k} \otimes A_{\ell}, A_{n} \otimes A_{m}\right)$-superbimodule in the natural way. Then we have

$$
\operatorname{Res}_{A_{k} \otimes A_{\ell}}^{A_{K}} \operatorname{Ind}_{A_{n} \otimes A_{m}}^{A_{K}} \cong{ }_{(k, \ell)}\left(A_{K}\right)_{(n, m)} \otimes-\text {. }
$$

On the other hand, for each $r \in \mathbb{N}$ satisfying $k-m=n-\ell \leq r \leq \min \{n, k\}$, we have

$$
\begin{gathered}
\operatorname{Ind}_{A_{r} \otimes A_{n-r} \otimes A_{k-r} \otimes A_{\ell+r-n}}^{A_{k} \otimes A_{2}} S_{23} \operatorname{Res}_{A_{r} \otimes A_{n-r} \otimes A_{k-r} \otimes A_{\ell+r-n}}^{A_{n} \otimes A_{m}} \cong M_{r} \otimes-, \quad \text { where } \\
M_{r}=\left(A_{k} \otimes A_{\ell}\right) \otimes_{A_{r} \otimes A_{k-r} \otimes A_{n-r} \otimes A_{\ell+r-n}} S^{23} \otimes_{A_{r} \otimes A_{n-r} \otimes A_{k-r} \otimes A_{\ell+r-n}}\left(A_{n} \otimes A_{m}\right),
\end{gathered}
$$

and where $S^{23}=A_{r} \otimes A_{n-r} \otimes A_{k-r} \otimes A_{\ell+r-n}$ (as an F-module) is a right $\left(A_{r} \otimes A_{n-r} \otimes A_{k-r} \otimes A_{\ell+r-n}\right)$ supermodule in the natural way and is a left $\left(A_{r} \otimes A_{k-r} \otimes A_{n-r} \otimes A_{\ell+r-n}\right)$-supermodule via the map

$$
\begin{gathered}
A_{r} \otimes A_{k-r} \otimes A_{n-r} \otimes A_{\ell+r-n} \rightarrow A_{r} \otimes A_{n-r} \otimes A_{k-r} \otimes A_{\ell+r-n}, \\
a_{1} \otimes a_{2} \otimes a_{3} \otimes a_{4} \mapsto(-1)^{\bar{a}_{2} \bar{a}_{3}} a_{1} \otimes a_{3} \otimes a_{2} \otimes a_{4} .
\end{gathered}
$$

Therefore, in order to prove the isomorphism (4.5), it suffices to prove that we have an isomorphism of superbimodules

$$
{ }_{(k, \ell)}\left(A_{K}\right)_{(n, m)} \cong \bigoplus_{r=n-\ell}^{\min \{n, k\}} M_{r} .
$$


For each $r \in \mathbb{N}$ satisfying $k-m=n-\ell \leq r \leq \min \{n, k\}$, define $w_{r} \in S_{K}$ by

$$
w_{r}(i)= \begin{cases}i & \text { if } 1 \leq i \leq r, \\ i-r+k & \text { if } r<i \leq n, \\ i-n+r & \text { if } n<i \leq n+k-r, \\ i & \text { if } n+k-r<i \leq K,\end{cases}
$$

Then the $w_{r}$ form a complete set of minimal length representatives of the double cosets in $S_{k} \times S_{\ell} \backslash S_{K} / S_{n} \times S_{m}$. If $C_{w}$ is the double coset containing $w_{r}$, then its cardinality is

$$
\left|C_{r}\right|=m ! n !\left(\begin{array}{l}
k \\
r
\end{array}\right)\left(\begin{array}{c}
\ell \\
n-r
\end{array}\right) .
$$

(See [SY15, proof of Prop. 4.3] for further details.) One easily verifies that

$$
w_{r}\left(a_{1} \otimes a_{2} \otimes a_{3} \otimes a_{4}\right)=(-1)^{\bar{a}_{2} \bar{a}_{3}}\left(a_{1} \otimes a_{3} \otimes a_{2} \otimes a_{4}\right) w_{r}
$$

for all $a_{1} \in A_{r}, a_{2} \in A_{n-r}, a_{3} \in A_{k-r}, a_{4} \in A_{\ell+r-n}$.

Define $M_{r}^{\prime} \subseteq{ }_{(k, \ell)}\left(A_{K}\right)_{(n, m)}$ to be the sub-superbimodule generated by $B^{\otimes r} \otimes w_{r}$. Then

$$
{ }_{(k, \ell)}\left(A_{K}\right)_{(n, m)}=\bigoplus_{r=n-\ell}^{\min \{n, k\}} M_{r}^{\prime}
$$

and $\operatorname{dim}_{\mathbb{F}} M_{r}^{\prime}=\left|C_{r}\right|\left(r \operatorname{dim}_{\mathbb{F}} B\right)$. Note that the dimension of $A_{k} \otimes A_{\ell}$ as a right supermodule over $A_{r} \otimes A_{n-r} \otimes A_{k-r} \otimes A_{\ell-n+r}$ is $k ! \ell ! / r !(n-r) !(k-r) !(\ell-n+r) !$ and that the dimension of $A_{m} \otimes A_{n}$ as a left supermodule over $A_{r} \otimes A_{n-r} \otimes A_{k-r} \otimes A_{\ell+r-n}$ is $m ! n ! / r !(n-r) !(k-r) !(\ell-n+r) !$. Therefore, $\operatorname{dim}_{\mathbb{F}} M_{r}=\left|C_{r}\right|\left(r \operatorname{dim}_{\mathbb{F}} B\right)=\operatorname{dim}_{\mathbb{F}} M_{r}^{\prime}$. Now consider the $\left(A_{k} \otimes A_{\ell}, A_{n} \otimes A_{m}\right)$-superbimodule map $M_{r} \rightarrow M_{r}^{\prime}$ uniquely determined by

$$
1_{A_{k} \otimes A_{\ell}} \otimes 1_{A_{n} \otimes A_{m}} \mapsto w_{r}
$$

which is well defined by (7.3). This map is surjective, and thus is an isomorphism by dimension considerations.

Lemma 7.4. We have an isomorphism of functors $\Psi^{\otimes 2} \Delta \Psi^{-1} \cong S_{12} \Delta$ on A-mod (hence also on A-pmod).

Proof. It suffices to prove that, for $m, n \in \mathbb{N}$, we have an isomorphism of functors

$$
\left(\Psi_{m} \otimes \Psi_{n}\right) \circ \operatorname{Res}_{A_{m} \otimes A_{n}}^{A_{m+n}} \circ \Psi_{m+n}^{-1} \cong S_{12} \circ \operatorname{Res}_{A_{n} \otimes A_{m}}^{A_{m+n}} .
$$

Note that, for $k \in \mathbb{N}, \Psi_{k}^{-1}=A_{k}^{\psi} \otimes_{A_{k}}$, where $A_{k}^{\psi}$ denotes $A_{k}$, considered as an $\left(A_{k}, A_{k}\right)$ superbimodule with the right action twisted by $\psi_{k}$. Similarly, $\Psi_{k}=A_{k}^{\psi^{-1}} \otimes_{A_{k}}-$. Thus, describing each functor in (7.5) as tensoring on the left by the appropriate superbimodule, it suffices to prove that we have an isomorphism of superbimodules

$$
\left(A_{m}^{\psi^{-1}} \otimes A_{n}^{\psi^{-1}}\right) \otimes_{A_{m} \otimes A_{n}} A_{m+n}^{\psi} \cong S \otimes_{A_{n} \otimes A_{m}} A_{m+n}
$$

where $S$ is $A_{n} \otimes A_{m}$ considered as an $\left(A_{m} \otimes A_{n}, A_{n} \otimes A_{m}\right)$-supermodule via the obvious right action and with left action given by $\left(a_{1} \otimes a_{2}, s\right) \mapsto(-1)^{\bar{a}_{1} \bar{a}_{2}}\left(a_{2} \otimes a_{1}\right) s$ for $s \in S$ and homogeneous $a_{1} \in A_{m}, a_{2} \in A_{n}$. 
For $k \in \mathbb{N}$, let $1_{k}$ denote the identity element of $A_{k}$ and $1_{k}^{\psi}$ denote this same element considered as an element of $A_{k}^{\psi}$ (and similarly, with $\psi$ replaced by $\psi^{-1}$ ). It is straightforward to show that the map between the superbimodules in (7.6) given by

$$
\left(1_{m}^{\psi^{-1}} \otimes 1_{n}^{\psi^{-1}}\right) \otimes 1_{m+n}^{\psi} \mapsto\left(1_{n} \otimes 1_{m}\right) \otimes 1_{m+n} .
$$

(and extended by linearity) is a well-defined isomorphism.

Lemma 7.5. We have isomorphisms of functors $\nabla \cong \nabla S_{12}$ and $\Delta \cong S_{12} \Delta$ on $A$-mod (hence also on $A$-pmod).

Proof. Let $m, n \in \mathbb{N}$ and define $w \in S_{m+n}$ by

$$
w(i)= \begin{cases}i+m & \text { if } 1 \leq i \leq n, \\ i-n & \text { if } n<i \leq m+n .\end{cases}
$$

Then we have $w s_{i}=s_{w(i)} w$ for all $i=1, \ldots, m-1, m+1, \ldots, m+n-1$. Now, let $S$ be $A_{m} \otimes A_{n}$ considered as an $\left(A_{n} \otimes A_{m}, A_{m} \otimes A_{n}\right)$-supermodule via the obvious right action and with left action given by $\left(a_{1} \otimes a_{2}, s\right) \mapsto(-1)^{\bar{a}_{1} \bar{a}_{2}}\left(a_{2} \otimes a_{1}\right) s$, for $s \in S$ and homogeneous $a_{1} \in A_{n}$, $a_{2} \in A_{m}$. So we have an isomorphism of functors $S_{12} \cong S \otimes-$. It is straightforward to verify that the map

$$
A_{m+n} \rightarrow A_{m+n} \otimes_{A_{n} \otimes A_{m}} S, \quad a \mapsto a w \otimes(1 \otimes 1),
$$

is an isomorphism of $\left(A_{m+m}, A_{m} \otimes A_{n}\right)$-superbimodules. It follows that $\nabla \cong \nabla S_{12}$. The proof that $\Delta \cong S_{12} \Delta$ is analogous.

Corollary 7.6. Towers of wreath product algebras are strong and compatible.

Proof. It follows immediately from Proposition 7.3, Proposition 4.5, Lemma 7.4, and Lemma 7.5 that towers of wreath product algebras are strong and dualizing. Since we saw in the proof of Proposition 7.3 that $\chi^{\prime}=\gamma^{\prime}=0$, they are also compatible.

Remark 7.7. The towers of wreath product algebras described in this section should yield categorifications of (quantum) lattice Heisenberg algebras. For instance, this can be seen in [CL12] for a particular choice of $B$. For a description of the (quantum) lattice Heisenberg algebras as Heisenberg doubles, see [RS, §§7, 8]. It would be interesting to work out the details of this categorification for general $B$.

\section{The tOWER OF NilCOXETER GRADED SUPERALGEBRAS}

In this section we specialize the constructions of Section 5 to the tower of nilCoxeter graded superalgebras of type $A$. We will see that we recover a categorification of the polynomial representation of the quantum Weyl algebra. Such a categorification was sketched in [Kho01, §4.1] (see Remark 8.8). Recall that $\mathbb{F}$ is an arbitrary field of characteristic not equal to two.

Definition 8.1 (NilCoxeter graded superalgebra). Define the nilCoxeter graded superalgebra with parameters $(d, \epsilon) \in \mathbb{Z} \times \mathbb{Z}_{2}$, denoted by $N_{n}^{d, \epsilon}$, to be the graded unital F-superalgebra with generators $u_{1}, \ldots, u_{n-1}$, such that $\left(\left|u_{i}\right|, \bar{u}_{i}\right)=(d, \epsilon)$, for $i=1, \ldots, n-1$, subject to the relations

$$
u_{i}^{2}=0 \text { for } i=1,2, \ldots, n-1 \text {, }
$$




$$
\begin{gathered}
u_{i} u_{j}=(-1)^{\epsilon} u_{j} u_{i} \quad \text { for } i, j=1, \ldots, n-1 \text { such that }|i-j|>1, \\
u_{i} u_{i+1} u_{i}=u_{i+1} u_{i} u_{i+1} \text { for } i=1,2, \ldots, n-2 .
\end{gathered}
$$

Since the ideal generated by all the $u_{i}$ is nilpotent, $N_{n}^{d, \epsilon}$ has, up to grading shift and isomorphism, one simple supermodule, which is one dimensional, and on which the generators $u_{i}$ all act by zero. We denote this simple supermodule, in degree zero, by $L_{n}$. Since it is one dimensional, it is of type M. By [Kle05, Prop. 12.2.12], the projective cover of $L_{n}$ is $P_{n}:=N_{n}^{d, \epsilon}$, considered as an $N_{n}^{d, \epsilon}$-supermodule by left multiplication.

The reason we need the factor of $(-1)^{\epsilon}$ in the relations of Definition 8.1 is to satisfy axiom TA2. Indeed, we have a map of graded superalgebras

$$
\begin{gathered}
\rho_{n, m}: N_{n}^{d, \epsilon} \otimes N_{m}^{d, \epsilon} \rightarrow N_{n+m}^{d, \epsilon} \\
u_{i} \otimes 1 \mapsto u_{i}, \quad 1 \otimes u_{j} \mapsto u_{n+j}, \quad 1 \leq i \leq n-1,1 \leq j \leq m-1 .
\end{gathered}
$$

Axiom TA1 is clearly satisfied, and TA3 holds since $N_{n+m}^{d, \epsilon}$ is a free left and right supermodule over $N_{n}^{d, \epsilon} \otimes N_{m}^{d, \epsilon}$ of rank $\left(\begin{array}{c}n+m \\ n\end{array}\right)$. Since, for all $n, \operatorname{dim} \operatorname{Hom}_{N_{n}^{d, \epsilon}}\left(P_{n}, L_{n}\right)=1$ and $L_{n}$ is of type $\mathrm{M}, \mathrm{TA} 4$ is also satisfied and $\mathbb{k}=\mathbb{Z}_{q, \pi}$.

For each $w \in S_{n}$, and two reduced expressions $w=s_{i_{1}} \cdots s_{i_{r}}=s_{j_{1}} \cdots s_{j_{r}}$, we have, by the relations in Definition 8.1 that $u_{i_{1}} \cdots u_{i_{r}}= \pm u_{j_{1}} \cdots u_{j_{r}}$. We can then choose a basis $\left\{u_{w} \mid w \in S_{n}\right\}$ of $N_{n}^{d, \epsilon}$ that satisfies the following property:

$$
u_{v} u_{w}= \begin{cases}\alpha(v, w) u_{v w} & \text { if } \ell(v)+\ell(w)=\ell(v+w), \\ 0 & \text { otherwise, }\end{cases}
$$

where $\ell(w)$ is the length of an element $w \in S_{n}$, and $\alpha: S_{n} \times S_{n} \rightarrow\{ \pm 1\} \subseteq \mathbb{F}^{\times}$is a nontrivial 2-cocycle when $\epsilon=1$ and is the trivial cocycle when $\epsilon=0$.

Lemma 8.2. We have that $N_{n}^{d, \epsilon}$ is a Frobenius graded superalgebra of degree $\left(-d\left(\begin{array}{c}n \\ 2\end{array}\right), \epsilon\left(\begin{array}{l}n \\ 2\end{array}\right)\right)$. The Nakayama automorphism is given on the generators by $\psi_{n}\left(u_{i}\right)=u_{n-i}$.

Proof. Define a trace map $\operatorname{tr}_{n}: N_{n}^{d, \epsilon} \rightarrow \mathbb{F}$ by

$$
\operatorname{tr}_{n}\left(u_{w}\right)= \begin{cases}1 & \text { if } w=w_{0} \\ 0 & \text { if } w \neq w_{0}\end{cases}
$$

We need to show that $\operatorname{ker}_{n}$ does not contain any nonzero left ideal. Let $I$ be such an ideal and $0 \neq a=\sum_{w \in S_{n}} a_{w} u_{w} \in I$. Choose an element $w^{\prime}$ of maximal length such that $a_{w^{\prime}} \neq 0$. Then $\operatorname{tr}_{n}\left(u_{w_{0}\left(w^{\prime}\right)^{-1}} a\right)=\operatorname{tr}_{n}\left( \pm a_{w^{\prime}} u_{w_{0}}\right)= \pm a_{w^{\prime}} \neq 0$, which contradicts the hypothesis. The degree of the algebra is simply the negative of the degree of $u_{w_{0}}$, which is $\left(d\left(\begin{array}{l}n \\ 2\end{array}\right), \epsilon\left(\begin{array}{l}n \\ 2\end{array}\right)\right)$ because the length of $w_{0}$ is $\left(\begin{array}{l}n \\ 2\end{array}\right)$.

Now, for all $w \in S_{n}$ such that $s_{i} w \neq w_{0}$, we have that $\operatorname{tr}_{n}\left(u_{i} u_{w}\right)=0=\operatorname{tr}_{n}\left(u_{w} u_{n-i}\right)$. In the case where $s_{i} w=w w_{0}=w s_{n-i}$, we have $u_{i} u_{w}=(-1)^{\epsilon \ell(w)} u_{w} u_{n-i}$. Hence, for all $w \in S_{n}$,

$$
\operatorname{tr}_{n}\left(u_{i} u_{w}\right)=(-1)^{\bar{u}_{i} \bar{u}_{w}} \operatorname{tr}_{n}\left(u_{w} u_{n-i}\right),
$$

and the statement about the Nakayama automorphism follows. 
Proposition 8.3. The tower $N_{n}^{d, \epsilon}$ is strong with twist $(\chi, d, \epsilon)$, conjugation $\Psi$ and shift $(\delta, \sigma)$, where $\chi^{\prime}(n, m)=n m$ for $n, m \in \mathbb{N}, \chi^{\prime \prime}=0, \Psi$ is given by the Nakayama automorphism (see Proposition 6.7), $\delta=\left(\delta_{n}\right)_{n \in \mathbb{N}}=\left(d\left(\begin{array}{l}n \\ 2\end{array}\right)\right)_{n \in \mathbb{N}}$ and $\sigma=\left(\sigma_{n}\right)_{n \in \mathbb{N}}=\left(\epsilon\left(\begin{array}{l}n \\ 2\end{array}\right)\right)_{n \in \mathbb{N}}$.

Proof. By Lemma 8.2 and Proposition 6.7, we see that induction is conjugate shifted right adjoint to restriction with shift $(\delta, \sigma)$. We have

$$
\kappa_{\delta}(n, m)=\delta_{n+m}-\delta_{n}-\delta_{m}=d\left(\begin{array}{c}
n+m \\
2
\end{array}\right)-d\left(\begin{array}{l}
n \\
2
\end{array}\right)-d\left(\begin{array}{l}
m \\
2
\end{array}\right)=d n m .
$$

Similarly, $\kappa_{\sigma}(n, m)=\epsilon n m$. Hence condition S1 is satisfied by choosing $\kappa(n, m)=n m$.

The verification of S2 is almost identical to its verification in the proof of Proposition 7.3. The only modification is that one takes $B=\mathbb{F}$ and replaces the superbimodule isomorphism (7.2) by a shifted version:

$$
{ }_{(k, \ell)}\left(N_{K}^{d, \epsilon}\right)_{(n, m)} \cong \bigoplus_{r=n-\ell}^{\min \{n, k\}} M_{r}\{d(n-r)(k-r), \epsilon(n-r)(k-r)\} .
$$

Similarly, the map (7.4) is replaced by the degree $(d(n-r)(k-r), \epsilon(n-r)(k-r))$ superbimodule map

$$
1_{N_{k}^{d, \epsilon} \otimes N_{\ell}^{d, \epsilon}} \otimes 1_{N_{n}^{d, \epsilon} \otimes N_{m}^{d, \epsilon}} \mapsto u_{w_{r}}
$$

The proof that this is a superbimodule isomorphism uses, instead of (7.3), the relation

$$
u_{w_{r}}\left(a_{1} \otimes a_{2} \otimes a_{3} \otimes a_{4}\right)=(-1)^{\epsilon(n-r)(k-r)\left(\bar{a}_{1}+\bar{a}_{2}+\bar{a}_{3}+\bar{a}_{4}\right)+\bar{a}_{2} \bar{a}_{3}}\left(a_{1} \otimes a_{3} \otimes a_{2} \otimes a_{4}\right) u_{w_{r}}
$$

for all $a_{1} \in N_{r}^{d, \epsilon}, a_{2} \in N_{n-r}^{d, \epsilon}, a_{3} \in N_{k-r}^{d, \epsilon}, a_{4} \in N_{\ell+r-n}^{d, \epsilon}$.

Lemma 8.4. We have an isomorphism of functors $\Psi^{\otimes 2} \Delta \Psi^{-1} \cong S_{12} \Delta$ on $N^{d, \epsilon}$-grmod, hence also on $N^{d, \epsilon}$-grpmod.

Proof. The proof is the same as [SY15, Lemma 4.4].

Recall that we are working over the ring $\mathbb{Z}_{q, \pi}$. For a positive integer $n$, we define the $q^{d} \pi^{\epsilon}$-integer $[n]:=1+q^{d} \pi^{\epsilon}+\cdots+\left(q^{d} \pi^{\epsilon}\right)^{n-1}$. We also define the $q^{d} \pi^{\epsilon}$-factorial $[n] !:=\prod_{i=1}^{n}[i]$ and the $q^{d} \pi^{\epsilon}$-binomial $\left[\begin{array}{l}n \\ k\end{array}\right]:=\frac{[n] !}{[k ! ! n-k] !}$. By convention, we set $[0]=0$ and $[0] !=1$. Notice that, for all $n, k \in \mathbb{N}$, we have $\left[\begin{array}{l}n \\ k\end{array}\right] \in \mathbb{Z}_{q, \pi}$. It is straightforward to verify that $\operatorname{grdim} N_{n}^{d, \epsilon}=[n]$ !.

Since $L_{n}$ and $L_{m}$ are one dimensional over $\mathbb{F}$, we have

$$
\operatorname{grdim}\left(N_{n+m}^{d, \epsilon} \otimes_{N_{n}^{d, \epsilon} \otimes N_{m}^{d, \epsilon}}\left(L_{n} \otimes L_{m}\right)\right)=\left[\begin{array}{c}
n+m \\
n
\end{array}\right] .
$$

Since $N_{n+m}^{d, \epsilon}$ has a unique one-dimensional simple supermodule $L_{n+m}$, this implies that, in the Grothendieck group, we have

$$
\left[\operatorname{Ind}_{N_{n}^{d, \epsilon} \otimes N_{m}^{d, \varepsilon}}^{N_{n+m}^{d, \epsilon}} L_{n} \otimes L_{m}\right]=\left[\begin{array}{c}
n+m \\
n
\end{array}\right]\left[L_{n+m}\right] .
$$

We also have the obvious isomorphism of projective graded $N_{n+m}^{d, \epsilon}$-supermodules

$$
P_{n+m}=N_{n+m}^{d, \epsilon} \cong N_{n+m}^{d, \epsilon} \otimes_{N_{n}^{d, \epsilon} \otimes N_{m}^{d, \epsilon}}\left(N_{n}^{d, \epsilon} \otimes N_{m}^{d, \epsilon}\right)=\operatorname{Ind}_{N_{n}^{d, \epsilon} \otimes N_{m}^{d, \epsilon}}^{N_{n}^{d, \epsilon}}\left(P_{n} \otimes P_{m}\right)
$$


We thus have isomorphisms of algebras

$$
\begin{gathered}
\mathcal{K}\left(N^{d, \epsilon}\right) \cong \mathbb{Z}_{q, \pi}[x], \quad\left[P_{n}\right] \mapsto x^{n}, \\
\mathcal{G}\left(N^{d, \epsilon}\right) \cong \mathbb{Z}_{q, \pi}\left[y_{1}, y_{2}, \ldots\right] /\left(y_{n} y_{m}-\left[\begin{array}{c}
n+m \\
n
\end{array}\right] y_{n+m}\right), \quad\left[L_{n}\right] \mapsto y_{n} .
\end{gathered}
$$

For the restriction, note that $\operatorname{Res}_{N_{n}^{d, \epsilon} \otimes N_{m}^{d, \epsilon}}^{N_{n+m}^{d, \epsilon}} L_{n+m}$ is a one-dimensional supermodule over $N_{n}^{d, \epsilon} \otimes$ $N_{m}^{d, \epsilon}$. Thus, it is has to be isomorphic to $L_{n} \otimes L_{m}$, and in the Grothendieck group we have

$$
\left[\operatorname{Res}_{N_{n}^{d, \epsilon} \otimes N_{m}^{d, \epsilon}}^{N_{d, \epsilon}^{d, \epsilon}} L_{n+m}\right]=\left[L_{n}\right] \otimes\left[L_{m}\right] \in \mathcal{G}(A) \otimes \mathcal{G}(A) .
$$

Note that $N_{n+m}^{d, \epsilon}$ is a free $\left(N_{n}^{d, \epsilon} \otimes N_{m}^{d, \epsilon}\right)$-supermodule (either on the left or on the right) of rank $\left(\begin{array}{c}n+m \\ n\end{array}\right)$. More precisely, we see that

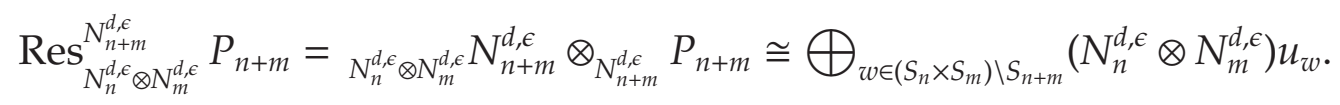

By considering the degrees of the basis elements $u_{w}$ of $N_{n+m}^{d, \epsilon}$ as an $\left(N_{n}^{d, \epsilon} \otimes N_{m}^{d, \epsilon}\right)$-supermodule, we see that, in the Grothendieck group,

$$
\left[\operatorname{Res}_{N_{n}^{d, \epsilon} \otimes N_{m}^{d, \epsilon}}^{N_{, \epsilon}^{d, \epsilon}} P_{n+m}\right]=\left[\begin{array}{c}
n+m \\
n
\end{array}\right]\left(\left[P_{n}\right] \otimes\left[P_{m}\right]\right) \in \mathcal{K}\left(N^{d, \epsilon}\right) \otimes \mathcal{K}\left(N^{d, \epsilon}\right) .
$$

We can summarize this discussion in the following proposition.

Proposition 8.5. There are isomorphisms of twisted bialgebras

$$
\mathcal{K}\left(N^{d, \epsilon}\right) \cong \mathbb{Z}_{q, \pi}[x] \text { and } \mathcal{G}\left(N^{d, \epsilon}\right) \cong \mathbb{Z}_{q, \pi}\left[y_{1}, y_{2}, \ldots\right] /\left(y_{n} y_{m}-\left[\begin{array}{c}
n+m \\
n
\end{array}\right] y_{n+m}\right),
$$

where the coproducts are given by

$$
\Delta\left(x^{n}\right)=\sum_{k=0}^{n}\left[\begin{array}{l}
n \\
k
\end{array}\right] x^{k} \otimes x^{n-k} \quad \text { and } \quad \Delta\left(y_{n}\right)=\sum_{k=0}^{n} y_{k} \otimes y_{n-k}
$$

Corollary 8.6. The tower $N^{d, \epsilon}$ is compatible.

Proof. By Proposition 8.5, $\mathcal{K}\left(N^{d, \epsilon}\right)$ is cocommutative, hence, by Lemma 8.4 and Proposition $4.5, N^{d, \epsilon}$ is dualizing, with a $\left(q^{d} \pi^{\epsilon}, 0, \kappa\right)$-twisted Hopf pairing, where $\kappa(n, m)=n m$ by $(8.3)$. In the proof of Proposition 8.3, we saw that $\mathcal{G}\left(N^{d, \epsilon}\right)$ is a $\left(q^{d} \pi^{\epsilon}, \kappa, 0\right)$-Hopf algebra; but since it is cocommutative, it is also a $\left(q^{d} \pi^{\epsilon}, 0, \kappa\right)$-Hopf algebra (see [RS, Rem. 2.4]). Hence (2.6) is satisfied, and so $N^{d, \epsilon}$ is a compatible tower.

The pairing (4.2) satisfies $\left\langle x^{m}, y_{n}\right\rangle=\left\langle\left[P_{m}\right],\left[L_{n}\right]\right\rangle=\delta_{m n}$. Therefore, $x^{*}\left(y_{m}\right)=y_{m-1}$ and so the twisted Heisenberg double $\mathfrak{h}=\mathfrak{h}\left(N^{d, \epsilon}\right)$ is the $\mathbb{Z}_{q, \pi}$-subalgebra of End $\mathbb{Z}_{q, \pi}\left[y_{1}, y_{2}, \ldots\right] /\left(y_{n} y_{m}-\right.$ $\left.\left[\begin{array}{c}n+m \\ n\end{array}\right] y_{n+m}\right)$ generated by $y_{1}, y_{2}, \ldots$ and $x^{*}$.

Notice that

$$
\mathcal{G}_{\text {proj }}(A) \cong \mathbb{Z}_{q, \pi}\left[y_{1}\right],
$$

and the Cartan map $\mathcal{K}(A) \rightarrow \mathcal{G}(A)$ of Definition 5.1 corresponds to the antilinear map

$$
\mathbb{Z}_{q, \pi}[x] \hookrightarrow \mathbb{Z}_{q, \pi}\left[y_{1}, y_{2}, \ldots\right] /\left(y_{n} y_{m}-\left[\begin{array}{c}
n+m \\
n
\end{array}\right] y_{n+m}\right), \quad x \mapsto y_{1} .
$$

Since

$$
\left\langle x^{m}, y_{1}^{n}\right\rangle=\left\langle\left[P_{m}\right],[n] !\left[L_{n}\right]\right\rangle=\delta_{m n}[n] !,
$$


we have $x^{*}\left(y_{1}^{n}\right)=[n] y_{1}^{n-1}$. For simplicity, define $y=y_{1}$. Then $x^{*}=\partial_{y}$ corresponds to the quantum partial derivation by $y$. Thus, we have an isomorphism of algebras

$$
\mathfrak{h}_{\text {proj }}=\mathfrak{h}_{\text {proj }}\left(N^{d, \epsilon}\right) \cong \mathbb{Z}_{q, \pi}\left\langle y, \partial \mid \partial y=q^{d} \pi^{\epsilon} y \partial+1\right\rangle \text {. }
$$

The Fock space $\mathcal{F}_{\text {proj }}$ is the representation of $\mathfrak{h}_{\text {proj }}$ given by its natural action on $\mathbb{Z}_{q, \pi}[y]$.

Remark 8.7. The algebras $\mathfrak{h}$ and $\mathfrak{h}_{\text {proj }}$ are closely related to the quantum Weyl algebra. In particular, when $\epsilon=0$, the numbers $[n]$ ! are not zero divisors and we have an isomophism

$$
\mathcal{G}(A) \cong \mathbb{Z}_{q, \pi}\left[x^{n} /[n] !\right], \quad y_{n} \mapsto x^{n} /[n] ! .
$$

In this case, $\mathfrak{h}_{\text {proj }}$ in an integral form of the rank one quantum Weyl algebra with parameter $q^{d}$.

Since the tower $N^{d, \epsilon}$ is strong and compatible, Theorem 5.7 provides a categorification of the polynomial representation of the quantum Weyl algebra when $\epsilon=0$ and $d=1$. More precisely, let $\mathbb{F}_{i}$ denote the trivial $N_{i}^{1,0}$-supermodule for $i=0,1$. Then (5.6) becomes

$$
\begin{aligned}
& \operatorname{Res}_{\mathbb{F}_{1}} \circ \operatorname{Ind}_{\mathbb{F}_{1}}=\bigoplus_{n}\left(\operatorname{Ind}_{A_{1} \otimes A_{n-1}}^{A_{n}} \circ \operatorname{Res}_{\mathbb{F}_{0} \otimes \mathbb{F}_{1}}^{\dagger}\left(\mathbb{F}_{1} \otimes-\right)\right) \oplus\left(\operatorname{Ind}_{A_{0} \otimes A_{n}}^{A_{n}} \circ \operatorname{Res}_{\mathbb{F}_{1} \otimes \mathbb{F}_{0}}^{\dagger}\left(\mathbb{F}_{1} \otimes-\right)\right) \\
& \cong \bigoplus_{n}\left(\operatorname{Ind}_{A_{1} \otimes A_{n-1}}^{A_{n}}\left(\operatorname{HOM}_{A_{0} \otimes A_{1}}\left(\mathbb{F}_{0} \otimes \mathbb{F}_{1}, S_{23}\left(\mathbb{F}_{1} \otimes \mathbb{F}_{0} \otimes \operatorname{Res}_{A_{n-1} \otimes A_{1}}^{A_{n}}(-)\right)\right)\right)\left\{\kappa(0,1)+\chi^{\prime \prime}(1-0,1), 0\right\}\right. \\
& \left.\quad \oplus \operatorname{Ind}_{A_{0} \otimes A_{n}}^{A_{n}}\left(\operatorname{Hom}_{A_{1} \otimes A_{0}}\left(\mathbb{F}_{1} \otimes \mathbb{F}_{0}, S_{23}\left(\mathbb{F}_{0} \otimes \mathbb{F}_{1} \otimes \operatorname{Res}_{A_{n} \otimes A_{0}}^{A_{n}}(-)\right)\right)\right)\left\{\kappa(1,0)+\chi^{\prime \prime}(1-1,0), 0\right\}\right) \\
& \cong\left(\operatorname{Ind}_{\mathbb{F}_{1}} \circ \operatorname{Res}_{\mathbb{F}_{1}}\right)\{1,0\} \oplus \operatorname{Id},
\end{aligned}
$$

which is the categorification of the defining relation $\partial x=q x \partial+1$ of the quantum Weyl algebra. Note that $\operatorname{Res}_{\mathbb{F}_{1}}=\bigoplus_{n} \operatorname{Res}_{A_{n}}^{A_{n+1}}$ and $\operatorname{Ind}_{\mathbb{F}_{1}}=\bigoplus_{n} \operatorname{Ind}_{A_{n}}^{A_{n+1}}$.

Remark 8.8. A categorification of the quantum Weyl algebra along the lines described here was outlined in [Kho01, §4.1]. However, while the main functor isomorphism [Kho01, Prop. 16] is correct, some of the other statements of [Kho01, §4.1] seem to be false. In particular, the shifted restriction functor defined there does not yield a coalgebra structure. This is a result of the fact that the shift on the restriction from $N_{n}^{d, \epsilon}$ to $N_{k}^{d, \epsilon} \otimes N_{n-k}^{d, \epsilon}$ is taken to be $n-k$. In order to obtain a coalgebra structure, one can either shift by zero (as presented above) or by $k(n-k)$ (see [RS, Prop. 5.1]). In addition, even with the correct shift, one does not obtain a Hopf algebra structure on the Grothendieck groups. Rather, one gets a twisted Hopf algebra.

\section{REFERENCES}

[BL09] N. Bergeron and H. Li. Algebraic structures on Grothendieck groups of a tower of algebras. J. Algebra, 321(8):2068-2084, 2009.

[CL12] S. Cautis and A. Licata. Heisenberg categorification and Hilbert schemes. Duke Math. J., 161(13):2469-2547, 2012.

[Kho] M. Khovanov. Heisenberg algebra and a graphical calculus. arXiv: 1009.3295 [math.RT].

[Kho01] M. Khovanov. NilCoxeter algebras categorify the Weyl algebra. Comm. Algebra, 29(11):5033-5052, 2001.

[Kle05] A. Kleshchev. Linear and projective representations of symmetric groups, volume 163 of Cambridge Tracts in Mathematics. Cambridge University Press, Cambridge, 2005. 
[Lam99] T. Y. Lam. Lectures on modules and rings, volume 189 of Graduate Texts in Mathematics. SpringerVerlag, New York, 1999.

[LS12] A. Licata and A. Savage. A survey of Heisenberg categorification via graphical calculus. Bull. Inst. Math. Acad. Sin. (N.S.), 7(2):291-321, 2012.

[LS13] A. Licata and A. Savage. Hecke algebras, finite general linear groups, and Heisenberg categorification. Quantum Topol., 4(2):125-185, 2013.

[RS] D. Rosso and A. Savage. Twisted Heisenberg doubles. Comm. Math. Phys. To appear, availabe at http://dx.doi.org/10.1007/s00220-015-2330-z.

[SY15] Alistair Savage and Oded Yacobi. Categorification and Heisenberg doubles arising from towers of algebras. J. Combin. Theory Ser. A, 129:19-56, 2015.

D. Rosso: Department of Mathematics and Statistics, University of Ottana, and Centre de Recherches Mathématiques, Montréal

URL: http://mysite.science.uottawa.ca/drosso/

E-mail address: drosso@uottawa.ca

A. Savage: Department of Mathematics and Statistics, University of Ottawa

URL: http://AlistairSavage.ca

E-mail address: alistair.savage@uottawa.ca 